\title{
The Prediction of the Lifetime of the COVID-19 Virus using Parameter-dependent New Mathematical Models
}

DR.K.SELVAKUMAR .. ( $\sim$ k_selvakumar10@yahoo.cpm)

Anna University Chennai University College of Engineering Nagercoil https://orcid.org/0000-0002$6122-3342$

\section{Research Article}

Keywords: Coronavirus , Crown , COVID-19 virus , Lifetime, Parameter dependent, Mathematical MOdels , Best model, Optimal model

Posted Date: May 13th, 2021

DOI: https://doi.org/10.21203/rs.3.rs-501782/v1

License: (c) (i) This work is licensed under a Creative Commons Attribution 4.0 International License.

Read Full License 


\title{
The prediction of the lifetime of the COVID-19 virus using parameter-dependent new mathematical models
}

\author{
K. Selvakumar *
}

Received: 06 May 2021

\begin{abstract}
This article is about a complex real-world human medical problem that people all over the world face, a major international public Health problem due to the new coronavirus disease 2019(COVID-19), a highly communicable infectious disease between humans. Spreads rapidly among humans of both sexes of all ages, in large masses in the cyclical manner(seasonally) causing disease in susceptible human Hosts affecting most of the organs in humans mainly lungs resulting in Severe Acute Respiratory Syndrome resulting in mass acute deaths. Acute deaths are more common with Comorbidities like Diabetes mellitus, Ischaemic heart disease, Liver disease, Kidney disease, Gut, etc. Now it is the major emergency international pandemic public health medical disease.

On the face of the earth, there are large masses of infection and mass acute deaths due to COVID-19 virus infection and so the life of every individual is uncertain at any time. Because of the mass acute deaths from the COVID-19 virus infection, everyone in the world is scared. From now on, it is the responsibility of the researchers of all nations to bring hope to people. In this article, by predicting the lifetime of diseasecausing virus, hope to the people is given, to better protect all people and speed up the immediate general pandemic preparedness within the lifespan of the virus.

To accelerate actions to save people's lives, mathematical models will help make public health decisions and reduce mortality using the resources available during this time of the COVID-19 pandemic. In this article, to better protect people from disease preparedness for the virus and a general pandemic by predicting the lifetime of the disease-causing coronavirus, three new mathematical models which are dependent on parameters are proposed. The parameters in the model function model uncertainty of death due to the present international real-life problem caused by different strains of

\footnotetext{
* Corresponding author

Dr. K. Selvakumar

Department of Science and Humanities, Anna University, Chennai, University College of Engineering, Nagercoil-629004. Tamilnadu, India

Tel.: +91-6380528672

E-mail: $k_{-}$selvakumar10@yahoo.com (selvakumaruce@gmail.com)
} 
the COVID-19 virus. The first model is a model with six parameters and the second and third models are models with seven parameters respectively.

These three models are the generalization of the three models of Phem [75]. The errors due to the models of this article are minimized from the errors due to the models of Phem. These three models can predict the acute death count outside the data period and can predict the lifetime. To illustrate the applicability of the models a big data set of size 54 days starting from February 29, 2020, to April 22, 2020, of acute death counts of USA( United States of America) is considered. The main focus is on the USA due to the significant large mass of infection and large mass of acute death from the COVID-19 virus. As a result, everyone's life is uncertain about death at any time.

Since it is a major international public health-related medical problem in humans, with an accuracy of $95 \%$ of confidence the results using three models are erected. The large mass of acute deaths due to the number of COVID-19 virus infections in the USA are fitted by the model functions of three mathematical models and a solution is found to an international problem. Based on the acute death rate, the lifetime of the COVID-19 virus is estimated to be 1484.76198616309920 days from the first day of acute death, February 29, 2020. In other words, there will be no mass acute deaths from the COVID-19 virus in the USA after April 2024 if the nation follows the guidelines of the WHO(World Health Organization) and the recommendations of the pathogen. And when the people and the government are very well prepared for this crisis then the spread of infection can be prevented, the people and government can be saved from the economic crisis, and many lives can be saved from mass acute deaths.

A comparative study of all models is presented for different measures of errors. The acute death count of the USA outside the date of the data set of 54 days is predicted using three models. The data set misses some counts during the collection of data and it is identified. From the ratio of standard deviation and average acute deaths, it is predicted that the total acute death counts during 54 days will be 62,969. Using the standard deviation around the line of regression it is shown that in the data set a large count is missing during the collection of data of USA. Using the coefficient of determination it is predicted that the Model-C, provides $100 \%$ of fitness with the given data set and only $0.0 \%$ variation. All three models are suitable to fit the data set of acute death counts of the USA, but Model-C is the best and optimal among the three models. Tt is predicted from Model-A, Model-B, and Model-C the total acute death counts during 54 days will be 66537, 67085, and 68523 respectively. Since Model-C is the best and optimal model, the predicted total acute death counts during 54 days will be 68523 .

Finally, this article suggests various steps to help control the spread and severity of the new disease. The prediction of the lifetime and data count missing in the data set presented in this research article is entirely new and differs totally from all other articles in the literature. To accelerate actions to save people's lives, mathematical models will help make public health decisions and reduce mortality using the resources available during this time of the COVID-19 pandemic. 
Keywords Coronavirus · Crown · COVID-19 virus · Lifetime · Parameter dependent $\cdot$ Mathematical M0dels $\cdot$ Best model $\cdot$ Optimal model

\section{Introduction}

In Latin, corona means crown. Coronaviruses $(\mathrm{CoV})$ on their surface have thorny protrusions look like crowns. And hence, viruses with crowns are termed as Coronaviruses. It is a large family of viruses found in birds like bats, and in animals like cats, cattle, and camels. Coronavirus from an animal can transmit the disease leading to a mass acute deaths to humans a new type of coronavirus can emerge(when germs from an animal are transmitted to a human). It can cause serious illness. This can be due to several factors, most notably people's lack of immunity to the new virus.

There are two ways that the disease can be passed on to humans. First, the virus from an animal must be ransmitted in to human. And, secondly, the virus entered the human must have potential to infect human. To enter the human body, the virus has to enter our cells, multiply, and should not be destroyed by our immune system. From this human to infect and spread to the other humans, the virus need to come out of the human body. There are common traits between animal hosts and humans. Perhaps the animal virus already shares one or more of these common characteristics. The virus undergoes genetic changes or mutations to achieve these properties either before it penetrates the whole human or during human-to-human transmission

In 2003, coronavirus(SARS-CoV) with crowns caused Severe Acute Respiratory Syndrome(SARS) identified for the first time. In 2012, coronavirus(MERS-CoV) with crowns caused a disease called Middle East Respiratory Syndrome(MERS) identified for the first time [122]. In 2019, coronavirus(SARS-CoV-2) with crowns causes Severe Acute Respiratory Syndrome identified for the first time in China.

The coronavirus SARS-CoV-2 infects people with the help of their crowns via a protein at its crown-shaped ends on the surface of the virus, hence the virus was called the corona. On the surface of human cells, the virus protein can interact with a human protein, allowing the virus to trap and infect human cells in our airways.

\subsection{Zoonotic ShifT and Zoonosis}

The process of an animal virus or bacteria infecting humans is called a zoonotic mutation. The disease caused by zoonotic virus is termed as zoonosis or zoonosis.
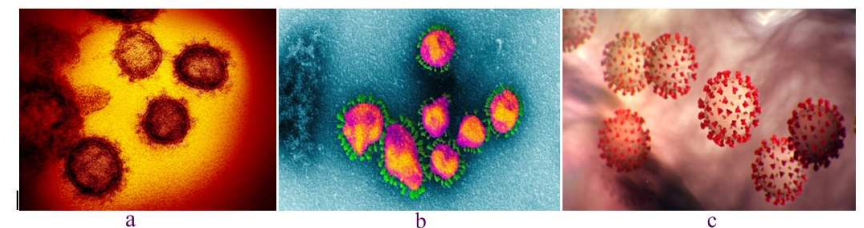

Fig. 1 a). SARS-CoV, b). MERS-CoV, and c). SARS-CoV-2 with crowns 
Examples of zoonotic viruses are HIV(non-human primates origin), Ebola virus(bats origin), SARS CoVs(bats origin), and avian influenza virus(bats origin ). Researchers suggest that this virus may be derived from bats, while other animals linked to infection in humans have not been confirmed. In [103], V'kovski reviewed the first, second, and third zoonotic movements of the coronavirus in the human community in 2003, 2009, and 2019. The infection of the coronavirus, its life cycle, and its connections to the biology of the coronavirus are examined. The differences and similarities between the three types of viruses are also examined. The threat posed by such a zoonotic change causes the current outbreak of the coronavirus originating in China, which is now officially declared as a pandemic by the WHO.

Ziqi Chen in [22] from Harward University explained the journey of the virus. The virus is believed to originate from the reservoir host (bats), and is transmitted to unconfirmed intermediate hosts (one suspected species is a pangolin). This virus have undergone undergone mutations that would have allowed it to pass from intermediate hosts to humans and then spread from one person to another person. The transmission and possible results of infection with the COVID-19 virus are given in [22].

In case of human coronavirus, the common cold is a symptom. But in case of animal coronavirus the common symptoms of the new COVID-19 are dry cough, fever, and fatigue. Recent noticed symptoms in some of the infected may include a sore throat, diarrhea, pain, nasal congestion, or a runny nose. Few people may get infected but don't show symptoms and feel uncomfortable. About $80 \%$ of people recover without special treatment from the disease. Around $20 \%$ of people infected is seriously ill and have difficulty in breathing. Older people and people with underlying diseases such as diabetes, heart problems, or high blood pressure tend to develop serious diseases indirectly in our society. According to the latest data, around 3-4\% of reported cases worldwide have died, but the rate of death changes with respect to age, the presence of underlying diseases, and location, Four types of human coronavirus are responsible in adults for 10 to $30 \%$ of the reliable sources of upper respiratory infections . [94, 126].

\subsection{First zoonotic movement of the coronavirus}

The first zoonotic movement of the SARS-CoV coronavirus began in 2003. Peiris et al., [61], review scientific advances in the study of the SARS-CoV virus. They also highlight advances in the development of therapies and vaccines. They developed a method for identifying and controlling future infectious disease threats. Stadler et al., in [96], present an overview of SARS. And they show that the 114-day SARS epidemic paralyzed the economy of Asia(29 countries struck , 8,098 people affected, 774 people killed). To attack the virus quarantine measures, notably by rising temperatures in summer, ended successfully and ensured the first SARS outbreak. The genomics of SARS-CoV, its phylogeny, its antigen structure, its immune response, and its possible therapeutic interventions are investigated if the SARS epidemic reoccurs., $[15,128]$. 
1.3 Second zoonotic movement of the coronavirus

The second zoonotic movement of the MERS-CoV coronavirus began in 2009. Algahtani et al., [6] presents an overview of MERS-CoV. A report of 2 cases is presented in this review. In Saudi Arabia in September 2012, MERS-CoV was first discovered. It infected more than 1,600 and resulted more than 580 deaths in laboratory tests. Infection of MERS-CoV is a serious disease that affects many complications of the lungs, kidneys, hematology, and gastrointestinal tract. In [6] the report of the neurological complaint in 2 adult patients due to MERS-CoV is reported and they set up the pathological hypothesis.

\subsection{The origin of the new COVID-19 virus in China}

The Wuhan Municipal Health Commission of China in Wuhan City, Hubei Province, reported a group of 27 cases of pneumonia of unknown etiology on December 31, 2019, including 7 serious cases associated with the fruit wholesaler. a living market ( Animals selling different species of animals). Clinical features in human is similar to that of infectious respiratory diseases such as shortness of breath, fever, and bilateral pulmonary infiltrate on the chest x-ray. The authorities isolated all cases, initiated contact tracing activities, and initiated sanitary and environmental hygiene measures in the market, which was closed to public use from January 1, 2020. The authorities of China had not reported any human transmission or health professional cases at the time.

The Chinese CDC On January 9, 2020 reported that 2019-nCoV(a novel coronavirus) is responsible for 15 out of 59 cases of pneumonia [129]. The first new sequence of the coronavirus genome was made available to the public [120] on January 10, 2020. The images were uploaded to the GenBank database and uploaded as part of the Global Influenza Data Sharing Initiative(GISAID) [127]. A preliminary analysis has shown that the new coronavirus clusters $(2019-\mathrm{nCoV})$ differ from the basic genome of known bat CoVs. During the period between, December 31, 2019, and January 20, 2020, nearly 295 confirmed cases reported to the laboratory, including 4 deaths [121]. Among the reported cases in Wuhan were 15 health care workers [6]. The confirmed cases 291 out of the 295 cases in laboratory are reported counts from Wuhan City, Guangdong, Beijing, and Shanghai as 270, 14, 5, and 2 respectively [129].

The Wuhan city reports that 169 cases are still under treatment in the hospital, including 35 seriously ill and nine seriously ill [134]. In Guangdong, 2 of the 14 reported cases did not travel to Wuhan, China, but came into contact with confirmed cases in the past [120]. The other four cases confirmed by the laboratory concern trips (1 from S outh Korea, 1 from Japan, and 2 from Thailand) [127, 130,135]. Of the 4 deaths reported in China, the first death occurred in a patient aged 61 on January 09, 2020, who visited for seafood, the wholesale market for Huanan seafood in Wuhan [130]. A second death occurred in a patient man with multiple organ failure aged 64 on January 15, 2020, [131]. The third death was reported on January 18, 2020 [133] , the fourth death occurred in a patient woman with coronavirus and pre-existing 
diseases aged 89 on January 19, 2020, [134]. During December 8, 2019, to January 18,2020 , more than half of the confirmed cases were men. In the reported cases, the age range is in between 10 and 89 years [120]. The history of other live markets in China is unknown till now for the recently reported cases [135]. In China, 1,739 patients have been identified as close contacts, and after continued follow-up, 817 patients have finished the observation period, the remaining 922 patients are under medical observation [131,132].

The WHO was notified of several cases of respiratory disease of unknown origin in the city of Wuhan $[119,124]$ in the Chinese province of Hubei on December 31,2019 , including clinical manifestations that resembled viral pneumonia and manifested as caugh, fever, and shortness of breath [118]. As of January 30, 2020, the WHO declared this epidemic as a global health emergency [18].

\subsection{Third zoonotic movement of the coronavirus}

The third zoonotic movement of the COVID-19 coronavirus began in 2019. Several patients with pneumonia of unknown cause were found in the Wuhan city of China in December 2019. Again, on January 7, 2020, the pathogen was identified as the new $\mathrm{CoV}$, which will later be referred to as the novel coronavirus 2019 (2019-nCoV) [125]. Genome sequencing has shown that the genetic sequence of COVID-19 is similar to that of the SARS-associated $\mathrm{CoV}$, and a unique medical approach to treating this disease is essential to detect and control the spread of the virus. Wang [104] presents one such approach to treating associated pneumonia in 2019 based on the unique charecteristics of the recently discovered virus and our experience with China CoVs at the West China Hospital in Chengdu. Adhikari et al., [2], are reviewing COVID-19 at the beginning of the period. The background to this review is that COVID-19 will be the cause of a respiratory disease outbreak in Wuhan of China, as of December 2019.

Tavakoli et al., in [98], said: At the beginning of 2020, China alerted WHO to a group of unusual pneumonia cases in Wuhan city. After much speculation, a new type of coronavirus was introduced as a COVID-19 pathogen and this virus cause human SARS-CoV-2. The wide and rapid spread of COVID-19 has created fear around the world. According to the WHO, the incubation period for infected patients is 2 to 10 days and the acute death rate in is $4.3 \%$. The mortality rate is higher in the elderly and patients with chronic lung disease, diabetes, high blood pressure, and chronic diseases, including those with coronary artery disease. The healthy death rate is less than $1 \%$.

Rabi et al. [79] provides a summary of current knowledge about the 2019-nCoV and the disease it causes. Alene and Yadeta in [5] study or control coronavirus infections caused by COVID-19, and prevent future events. Razvan Azamfirei in [4] presents an overview of the coronavirus caused by COVID-19. An overwhelming sense of panic has penetrated the public discourse since the identification of the new 2019 coronavirus in the month of December 2019. This should be reinforced by the recent WHO statement on the novel coronavirus epidemic as an internationally worrying public health emergency. This is the third major occurrence of zoonotic $\mathrm{CoV}$ 
transmission that has crossed the species barrier to human infection and is likely not the last.

Chung et al., [18],contacted 21 patients with a history of human exposure from the endemic center of Wuhan, China, and analyzed and presented their results. Chung et al., [18], suggest that the lungs can react and can be healed similar to SARS and MERS, although it's too early to have picture descriptions for 2019 patient population. Song et al., [79] reported the results of 50 of 51 patients with a history of contact with people from Wuhan, China. Singapore has well-developed protocols in preparation for the COVID-19 outbreak. Cleland et al. [24] commented on precautionary measures to minimize the risk of virus transmission in Singapore. COVID 19 is spreading faster, health system weakness is not a vector of transmission for health workers with the worst prevention and control practices. Jackson et al., [37], evaluated this fact in Tanzania.

\subsection{Literature review of mathematical models}

To accelerate actions to save people's lives, mathematical models will help make public health decisions and reduce mortality using the resources available during this time of the COVID-19 pandemic.

Artificial intelligence(AI) techniques such as fuzzy logic(FL), neural networks(NN), and evolutionary computing(EC) can be applied to discuss COVID-19 data and predict outcomes that will be useful in saving the lives of the world's population. This subsection conducts a review of the literature on mathematical models related to AI techniques such as NN(Neural Network), FL(Fuzzy Logic), EC, deep learning(DL), and other related fields.

In [17], Choisy et al., the importance of epidemiological models is suggested. In [60] an overview of predicting COVID-19 disease and drug development using AI. In [38] AI and DL-GANA(Generative Adversarial Networks) methods, ELM(Extreme Learning Machne) and LSTM(Long-Short Term Memory) methods are used to predict the results in COVID-19. In [34] to predict the growth area of new-infected cases, deaths, and new cases cured in China and the US, ENN(Elman NN), LSTM, and SVM Carrier Vector Machine is used. SVM with fuzzy granulation is also used. In [3], the number of confirmed, recovered, and fatal cases were predicted from July 11, 2020 to July 17, 2020 using an ANN(Artificial NN) using the Pakistani dataset from February 25 to July 10, 2020. In [25] India's future is predicted using SNN(Statistical $\mathrm{NN}$ ) models and their version. In [26] the Quantum NN(CQNN) is used to predict the severity of the disease COVID-19 in patients. In [30] a GRNN (Generalized Regression Network) model optimized with FPA(Flower Pollination Algorithm) is developed to predict the epidemic pattern. To predict the COVID-19 outbreak, in [33] use $\mathrm{AI}$ and DL with time series using a non-linear regressive network(NAR). In [58] the COVID-19 epidemic was predicted using ANN-based prediction models. In [97] a curve fitting based on ANN is used to predict the number of deaths from COVID19. In [99] an intelligent surveillance system is used to monitor people using deep CNN(Convolutional NN) models to prevent the spread of COVID-19. In [7], Ali et al., using the Machine Learning Applications Predict and Compare Spread Rates, In- 
fection Rates, and Mortality Rates with Other Pandemics, collected data on COVID19 patients and, in particular, information on the first deaths in 186 countries from November 17, 2019, to May 16, 2020. In [91] Shinde et al. conducted a study of forecasting techniques such as stochastic models and machine learning techniques by classifying real data. In [52] Mohamadou et al. did a review of AI and modeling and insists on exploring more models to control COVID-19. In [32], Fokas et al. designed a model for studying the epidemic situation in countries such as Spain, Germany, the USA, France, and Sweden. In [85] Santra designed a model that does not adapt itself directly, but indirectly to data by perturbing the data. In [1], Adiga et al., a review of the mathematical models is made that will be useful to researchers.

To control the spread of COVID-19, we are investigating in [9] how deep learning plays an important role. In [32], using the math expression and deep learning network, the number of infected cases was predicted in six countries, the USA, Germany, Italy, Spain, France, and Sweden, from the time the epidemic developed. In [77], a networkbased model, the Network Inference-Based Prediction Algorithm(NIPA) is used to predict the future course of the epidemic in all cities in Hubei Province in China. . The network consists of cities and their interactions in Huban Province. An exact prediction of the epidemic is noted. In [74] AI and Big Data are used to improve the COVID-19 situation.

In [59], a Bayesian optimization framework for predicting the risk category of a country. It is a flat neural network based on the LSTM. To predict the future model of COVID infection, fuzzy time series(FTS) and ANN are usedin [51] and compared them with the ARIMA model using the data set from March 17, 2020 to July 1, 2020. In [53], the cumulative of the COVID-19 incidence rates across the country are predicted using the MLP(multilayer perceptron) neural network. Use an exponential model and machine learning in [42]. The first signs of COVID-19 can be seen by March 26, 2020. In [64] an ARMA model is used to monitor the spread of the epidemic in Italy, Russia, and the USA.

To predict the medical and economic factors due to the pandemic, [57] estimates the epidemic characteristics using the SIR (susceptible to infected withdrawal) model. In [100] the SIR model is used to estimate the cost of the management strategy. In [101] a mathematical SIR model with a dynamic density function is used for the spread of the disease. In [111] a stochastic mathematical SIR model for COVID-19 is developed to determine the spread of the disease control value.

In [44] a deep learning $\mathrm{CNN}$ is used to classify images of the infected lungs by chest $\mathrm{X}$-ray(CXR). In [82], CNN models and logistic regression(LR) are used to classify CXR images. In [81] a neural network is used for an unbalanced data set to detect cases of COVID-19. In [78], CXR images of COVID-19 of pneumonia and healthy patients are classified using deep neural networks. In [73], cases of COVID19 infected were predicted with computed tomography(CT) images using AI and CNN methods. In [105], patients with COVID-19 were detected from CXR images using deep CNN.

In [36], CNN architectures are used to detect COVID-19 disease from two sets of CXR images. In [48] information on predicting a fatal outcome in virus infected patients with COVID-19 using an ANN provided. In [49] CNNs were used to identify infected patients based on CXR images. In [88], patients with COVID-19 were 
recognized by training classifiers for deep learning and machine learning based on their CXR images. In [93] a deep CNN includes the identification of the COVID19 infection based on the CXR image of the patient's lungs to save doctors time in diagnosis.

To study the March 1, 2020 dynamics in India, mathematical models were used to fit the infected case record. And predicted future infection in India [13]. In [14] a mathematical model of the transmission dynamics is supposed to investigate the development of the epidemic. In [43] a discrete mathematical model for the dynamics of the transmission of humans and animals in different regions is designed. In [63] a mathematical model for the prediction of the infection dynamics in Brazil is examined. In [80] a traditional mathematical model of the spread dynamics in New York State is used as a prediction for an infection. In [107] a mathematical model of the dynamics of disease transmission in Ukraine is analyzed. In [109] a mathematical model is designed that uses the insulation class to predict the dynamic behavior of disease infection. In [110] a stochastic model for the dynamics of the unique transmission of the disease in mainland China is designed and the epidemic will be detected in and around mainland China in early March 2020. In [114] an epidemic model is introduced to estimate the unknown data in China. In [115], a mathematical model for total death in Asia Nations are proposed.

In [21] a mathematical model is supposed to predict the number of COVID-19 cases in China, Austria, Poland, and France. In [22] a mathematical model is used to calculate the transmission of the disease in a population from an infected population. In [29] an overview of COVID-19 from different databases is given. A review of the AI models for the drug COVID-19 is being carried out in [113]. In [35] mathematical models of infectious diseases that spread in the population are checked and applied to some diseases. During this lockdown, [50] developed a model to determine the risk of transmission and infection of COVID-19, and after the lockout at the time of business entry to determine the net profit.

In [12] three models are analyzed to predict and access the cause of the epidemic by region. In the absence of a vaccine, the effects of taxation and the risk of easing social distancing are discussed. In [8] an iterative method based on the Euler method and cubic spline interpolation is investigated to predict values from the begining of June 01, 2020, using data from February 15, 2020, to May 31, 2020. In [56] to predict cases infected in mainland China on February 10, 2020, a mathematical model is used. In [62] an iterative method is used to predict the daily growth rate by giving the input values the number of limited observations. In [84] mathematical models are given that predict the spread patterns of the COIVID-19 epidemic to better understand the spread.

A review of the AI models for the drug COVID-19 is being carried out in Zhu. In [108] the infection in Morocco is estimated and predicted using a mathematical model. A modified compartmental model for the spread of the disease in Morocco is introduced in [90] and strict home containment is observed to play an important role in controlling the spread. In [76] a logistic function inspired by the renormalization group is used to analyze data from infected cases from nations in the first phase. In [83] the cough airflow predicts the range of infectious droplets to a target that the mouth emits during a cough. In [87], action is being taken in Brazil to deal with 
the pandemic situation and a report will be presented. In [23] an overview of the pandemic is given for future preparation. An overview of the predictive models for diagnosing COVID-19 in patients is given in [106].

It is predicted that $40 \%$ to $70 \%$ of the world population in [55] will be infected in the coming years. In [65] a continuation of the COVID-19 coronavirus is predicted based on a sample. In [54] the effects of life expectancy are discussed. In [123] the use of the Susceptible Exposed Infectious Recovered(SEIR) calculation method forecast COVID-19 trends and plotted possible outcomes for the period from September 22, 2020, to February 28, 2021, using the COVID-19 infected case and mortality data from February 01, 2020, through September 21, 2020. Time series are used to analyze each state in the United States. In [40,41], mortality from COVID-19 is estimated to be less than one million deaths, and the remaining life of the population of the United States is observed to be reduced by less than a part in a thousand. COVID-19 claimed lives in months but not decades like other epidemics like VIHAIDS and opioids.

[39] discusses the importance of mathematical models for making public health decisions and reducing mortality using available all possible resources during the present COVID-19 pandemic situation. However, no mathematical expression is given in [39]. In [30], using a relationship between the spread of COVID-19 and weather parameters, Indian high-risk countries were predicted using the United States' forecast model. Singh et al., [92], forecast the spread of COVID-19 in terms of several confirmed cases, deaths, and recoveries from April 24, 2020 to July 7, 2020 using the ARIMA -Model For the hardest-hit countries, 15 countries ranked first in the world.

In [11] an excessive number of deaths over a year is recorded at various levels of COVID-19 transmission. In [28] the mortality rate for Eastern European countries is estimated. In the United States, the CDCP Centers for Disease Control and Prevention urges people to stay home when they are sick, avoid touching their nose and mouth while covering them, and frequently use them to shut their hands to wash. Soap before and after touching an object to prevent it from spreading of the coronavirus. [116]. In [89] the lifetime of the coronavirus in the USA using the logistic mathematical models is given.

The research, mathematical models, and life predictions presented in this article are completely new and different from any other article in the literature.

\subsection{Motivation of this work}

To accelerate actions to save people's lives, mathematical models will help make public health decisions and reduce mortality using the resources available during this time of the COVID-19 pandemic. Knowing how long this infection lasts in the country can help the government and voluntary organizations make public health decisions and reduce mortality. The work of Nicholas et al., [39], motivated me to do this work to determine the lifetime of the COVID-19 coronavirus and save lives. The daily news of mass acute deaths around the world and data on 215 countries, as well as Phon's [75] mathematical logistic model to predict the maximum number of mass acute deaths in the USA from COID-19 in the coming days, motivates to find the lifetime of the COVID-19 coronavirus(the point of no mass acute death from COVID-19). For bet- 
ter immediate prevention and preparation to face the situation, the lifetime of the COVID-19 virus can be calculated using logistic mathematical models. These models can contain information about the population of an area. Actual preparation for a COVID-19 pandemic will depend on actual cases in the population, whether or not they have been identified, said Srinivasa Rao of the University of Augusta. In the United States, with better numbers, we can better estimate how long the COVID-19 virus will last and how much it will worsen. Without these numbers, how can health systems and health workers prepare for what is needed? Said Rao. Better numbers are also important to better protect people and speed up the general pandemic preparedness [117]. This motivates to do this work to predict and estimate the lifetime of the COVID-19 virus.

\subsection{Main Results of this Article}

We want to better protect people from the disease-causing virus COVID-19 and to speed up immediate general COVID-19 pandemic preparedness by predicting the lifetime of the disease-causing virus COVID-19 using three new logistic mathematical models that depend on parameters. The parameters of the model work with the uncertainty of the model due to the current international real health problem caused by the coronavirus. The first model is a six-parameter model, and the second and third models are seven-parameter models. Three models of Phem [75] are the particular cases of the models presented in this article. The errors due to the models in this article are minimized when compared to the errors due to the models in Phem [75]. These three models can predict the number of mass acute deaths out-of-date mass acute deaths and lifetime.

A comparative study of all models is presented for different measures of error. The number of mass acute deaths in the United States outside the date of the 54-day data set is predicted using three models. The record is missing some counts during data collection and is identified. From the ratio of the standard deviation to the mean value of the deaths, a total of 62,969 deaths is forecast for 54 days. The standard deviation around the line regression shows that a large number is missing from the data set when collecting data from the United States. Using the coefficient of determination, it is predicted that the Model-C will provide $100 \%$ fitness with the given data set and only $0.0 \%$ variation. All three models are suitable for the US dataset, but Model-C is the best and optimal of the three models. Based on Model-A, Model-B, and ModelC, the total acute deaths over 54 days are $66,557,67,085$, and 68,523, respectively. Since Model-C is the best and most optimal model, the total number of acute deaths predicted for 54 days will be 68,523 . In this article, the main findings are the prediction of the life span of the coronavirus COVID-19 (the time without mass acute death from COVID-19) in the US using three mathematical models. Based on the total number of acute deaths at time $t$, the first, second, and third models predict COVID19 coronavirus lifetimes of 240.78947368421052 days, 240.35306157894738 days, and 274.77876106194691 days, respectively, from February 29, 2020. Based on the count of the best and optimal model, Model-C, three models predict that the life span of the COVID-19 coronavirus will be 274.77876106194691 days from the first day 
of acute death, February 29, 2020, that is, after 274.77876106194691 days from the first day of acute death, from February 29 2020., there will be no mass acute deaths and compared to the mass acute deaths from the WHO live updates, there will be an acute death from COVID-19 in the US even after December 2020.

Based on the acute death rate, the three mathematical models predict the lifespan of the COVID-19 coronavirus will be 1285.1211233845768 days, 1281.3377741935484 days, and 1484.7619861630992 days, respectively, from the first day of acute death, from February 29, 2020. The best and optimal model, Model-C predicts the life span of the new COVID-19 coronavirus as 1484.76198616309920 days from the first day of acute death, February 29, 2020. That is. after 1485 days from the first day of acute death, February 29, 2020 (that is, after April 2024) there will be no mass acute death from the new COVID-19 coronavirus if everyone follows WHO guidelines and advice from health workers. To complete this article, examines the origin of the coronavirus, the types of zoonotic movements of the coronavirus, and the transmission of the virus from bats to humans. This article describes how a COVID-19 virus spreads from an infected to an uninfected patient, that is, how a COVID-19 virus enters into the human lungs through the atmospheric air.

In brief, this article uses the best and optimal model, Model-C, to predict the life span of the COVID-19 coronavirus in the US as 1484.76198616309920 days from the first day of acute death, February 29, 2020. In other words, we cannot expect any mass acute deaths in the US from COVID-19 after April 2024, provided everything in the world follows WHO and the guidelines Advice from health workers.

\subsection{Structure of this article}

In Section 1, the virus with a crown, zoonotic shift, zoonotic disease, and first, second, and third zoonotic movements of the coronavirus, followed by a survey of the mathematical models are presented. Section 2 shows how a COVID-19 virus spread from an infected patient to an uninfected patient. In Section 3, a seven-parameter dependent mathematical model for the new COVID-19 virus is derived that predicts the maximum number of mass acute deaths from COVID-19 in the coming days. From this model, we derive models with six parameters and two to seven parameters. Using the USA data set, parameters are obtained using the least-squares method. Section 4 calculates the lifespan of the COVID-19 coronavirus in the United States using a mathematical model, Model-A. Section 5 calculates the lifespan of the COVID-19 coronavirus in the United States using a mathematical model, Model-B. Section 6 calculates the lifespan of the COVID-19 coronavirus in the United States using a mathematical model, Model-C. Section 7 presents a comparative analysis of the proposed models with other models. Section 8 discusses the prediction of total acute deaths based on the United States dataset. Finally, Section 9 suggests rapid steps to control the rapid spread of the coronavirus and the severity of the disease, as well as a research plan for the COVID-19 coronavirus.

In the coming sections, the key findings of this article, the lifetime of COVID-19 in the US will be calculated to better protect people and to speed up the immediate general pandemic preparedness using logistic mathematical models. 


\section{The spread of COVID-19 in humans through contaminated atmospheric air}

This section introduces the transmission of a COVID-19 virus from an infected patient to an uninfected patient. The routes of entry into the lungs, such as how the COVID-19 virus enters the human lungs primarily through air contaminated with COVID-19 in the atmosphere, are discussed in the remainder of this section.

Respiratory secretions, which form as droplets and appear when an infected patient coughs, sneezes, yawns, or speaks, which contain the COVID-19 virus and are the primary means of the virus transmission (see Figure 2). There are two ways people can spread the disease COVID-19. The COVID-19 infection can spread to people within a meter of droplets that are spat out or exhaled by a patient with COVID-19. or humans can become infected through their unknown contact with contaminated surfaces or objects nearby and finally touching the infected secretions from your eyes, nose, or mouth. A person can touch the COVID-19 virus on a doorknob or their hands and then touch the face, nose, and mouth. For this reason, when disinfecting the door handle, hands, and the environment, handshaking is avoided, which is important. Current results show that the virus transmission can begin immediately. before symptoms appear. Regardless, many of those who have been infected with COVID-19 have mild symptoms in the early stages of the disease. It is believed that it is possible to catch COVID-19 from an infected person who has it. For example just a light cough and not feeling bad. The WHO is evaluating ongoing research on the transmission period of COVID-19 and continues to provide updated results [16] .

\section{Parameter-dependent mathematical model for the new COVID-19 virus}

This section derives a mathematical model that depends on six parameters to predict the maximum number of mass acute deaths in the US from COVID-19 in the coming days. Five and six parameter models are derived from this model. Using the US dataset, parameters are obtained using the least-squares method. A comparison of these three models is made using the US dataset to select the best model. A compari-

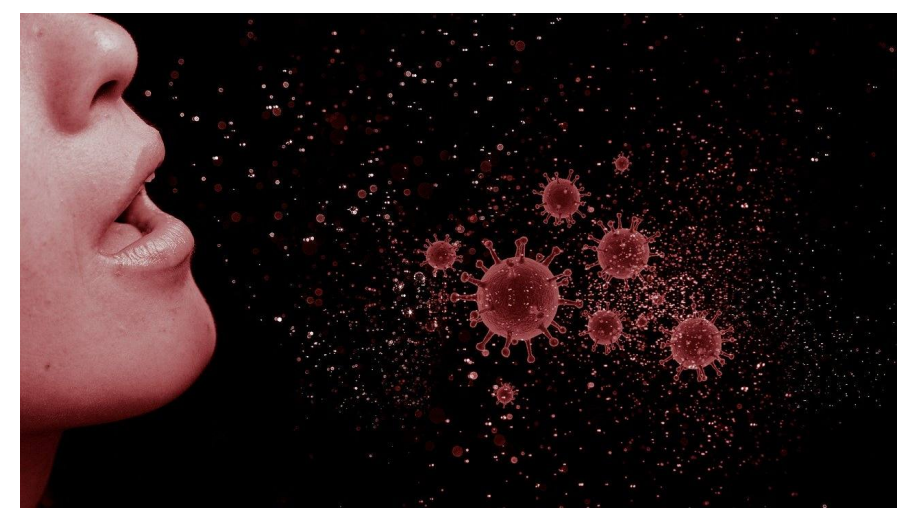

Fig. 2 The spread of COVID-19 virus from an infected patient. 
son of the predicted data from the United States using the proposed models is made. A comparison of the [75] models is also carried out. A comparison of the predicted US data using [75] models is presented. The number of mass acute deaths in the United States outside the date of the 54-day data set is predicted using three models.

A time-dependent function that estimates the total number of mass acute deaths in the population from COVID-19 is presented in [75]. The total number of mass acute deaths at time $t$ in the US is estimated using this model. The function corresponds to a data record from the USA. The [75] model predicts the maximum limit of the total number of mass acute deaths in the US in terms of time in days. The lifespan is not predicted in [75] and we have tried to estimate the lifespan of the infection that the COVID-19 virus causes.

The US mass acute death record from COVID-19 follows an S-shaped trajectory. This prompts us to turn to S-shaped logistics models. In the literature we have some S-shaped logistics models $[4,47,66-71,86,95,102,112]$ and logistic regression models [72]. Among these models are the logistics models designed by Pham [69] to estimate the number of errors. In [75] the model in [69] is changed by adding unknown parameters so that it includes the uncertainty. These parameters take into account the uncertainty of the acute deaths due to COVID-19 virus in population samples for different age groups, and different areas and environments. The optimal and the best model is selected based on a new Phan model selection criterion, which is called the Pham criterion(PC) in [75] and is compared with other existing criteria. In short, [75] develops a model for calculating the cumulative number of mass acute deaths in the population. The mass acute death from COVID-19 was included in the study with the assumption

1. Already when there are some in the population who have a COVID-19 infection and can spread the virus in a community. But not sure that the community is infected or not. The possibility of the viral infection spreading to people through close contact or inhalation directly into the lungs, touching their nose, mouth or eyes, or touching infected objects or surfaces. [123].

2. The spread of the virus over an entire area over time spread to a small number of people at a slow rate of infection and a large number of people at a higher rate. This infection rate will continue to increase until a maximum number of mass acute deaths is reached.

3. In the time-critical population $t$, the rate of change in acute death is proportional to the number of mass acute deaths of infected and non-infected people.

4. The mass acute death data is reliable as reported case data, hospital data for testing, and symptom and treatment data. Also, it is easy to identify the cause of mass acute death rather than the cause of life for hospitalizations and testing.

In the literature [66-70,72,102,89], models of population growth and mortality are applied to various past and present situations. The growth equation for the mass acute death results from the differential equation $[69,70,75]$,

$$
d^{\prime}(t)=q(t) d(t)[s-d(t)], t \geq 0,{ }^{\prime}=\frac{d}{d t}
$$

where $d(t), q(t)$ and s relate to the cumulative acute mortality at time $t$, the acute mortality rate and the maximum number of the mass acute deaths, respectively. The 
solution of the ordinary differential equation (1) has the form

$$
d(t)=\frac{s}{1+C \exp \left(s \int_{0}^{t} q(t) d t\right)}, C=\frac{s-d(0)}{d(0)} .
$$

To improve the required goodness of fit with the data set, take $\mathrm{q}(\mathrm{t})=\mathrm{q}>0$ and the approximation function (2) given the uncertainty of the COVID coronavirus -19 in the population by adding more parameters. We reformulate the function (2) as

$$
d(t)=\frac{s}{1+C \exp (-s q t)}=\frac{s}{1+C \frac{1}{\exp (s q t)}}=\frac{s}{1+C\left[\frac{1}{\exp (q t)}\right]^{s}} \approx \frac{s}{1+C\left[\frac{1+p}{r+\exp (q t+v)}\right]^{s}} .
$$

Here $\mathrm{v}$ is a constant parameter. For goodness of fit, again approximate as follows,

$d(t) \approx \frac{s}{1+C\left[\frac{1+p}{r+\exp (q t+v)}\right]^{s}} \approx \frac{s}{1+C\left[\frac{1+p}{r+\exp (q t+v)}\right]^{s-n} \cdot\left[\frac{1+p}{r+\exp (q t+v)}\right]^{n}} \approx \frac{s}{1+k\left[\frac{1+p}{r+\exp (q t+v)}\right]^{n}}$

where $\mathrm{k}=\mathrm{C}\left[\frac{1+p}{r+\exp (q t+v)}\right]^{s-n}$.

In order to improve the best fit to the data set, we reformulate the function (2) taking into account the assumptions and the cumulative number of the mass acute deaths at time $\mathrm{t}$

$$
d(t)=\left[\frac{s}{1+k\left[\frac{1+p}{\exp (q t+v)+r}\right]^{n}}\right], t \geq 0
$$

where $\mathrm{p}, \mathrm{q}, \mathrm{r}, \mathrm{s}, \mathrm{k}, \mathrm{v}$, and $\mathrm{n}$ are seven parameters related to the number of the mass acute deaths in the United States for 54 days from February 29, 2020 to April 22, 2020 using the least squares method are determined. In addition, the function fulfills the hypotheses

1. At the beginning of the infection at $t=0$ there are some in the population who have a COVID-19 infection, and therefore the function (5) takes on the value [123].

$$
d(0)=\left[\frac{s}{1+k\left[\frac{1+p}{\exp (v)+r}\right]^{n}}\right] \neq 0 .
$$

2. As time progress, $t \rightarrow \infty, \mathrm{d}(\infty)=\mathrm{s}$, from the data set of USA.That is,

$$
\lim _{t \rightarrow \infty} d(t)=s
$$

The function $(5)$ with $d(0) \neq 0$ is considered a new logistic function in this article

$$
d(t)=\left[\frac{s}{1+k\left[\frac{1+p}{\exp (q t+v)+r}\right]^{n}}\right], t \geq 0, d(0) \neq 0 .
$$

This is a seven parameter model, and the seven parameters of the function (8) can be estimated using the least-squares method. 
Table 1 Data set of USA.[75,89]

\begin{tabular}{llllllllll}
\hline Day & Counts & Day & Counts & Day & Counts & Day & Counts & Day & Counts \\
\hline Feb 29 & 1 & March 01 & 01 & 02 & 06 & 03 & 09 & 04 & 11 \\
05 & 12 & 06 & 15 & 07 & 19 & 08 & 22 & 09 & 26 \\
10 & 30 & 11 & 38 & 12 & 41 & 13 & 48 & 14 & 58 \\
15 & 73 & 16 & 95 & 17 & 121 & 18 & 171 & 19 & 239 \\
20 & 309 & 21 & 374 & 22 & 509 & 23 & 689 & 24 & 957 \\
25 & 1260 & 26 & 1614 & 27 & 2110 & 28 & 2754 & 29 & 3251 \\
30 & 3948 & 31 & 5027 & April 01 & 6263 & 02 & 7438 & 03 & 8694 \\
04 & 10,231 & 05 & 11,632 & 06 & 13,128 & 07 & 15,347 & 08 & 17,503 \\
09 & 19,604 & 10 & 21,830 & 11 & 23,843 & 12 & 25,558 & 13 & 27,272 \\
14 & 29,825 & 15 & 32,443 & 16 & 34,619 & 17 & 37,147 & 18 & 39,014 \\
19 & 40,575 & 20 & 42,514 & 21 & 45,179 & 22 & 47,520 & & \\
\hline
\end{tabular}

3.1 Three mathematical models depending on the parameters of the seven-parameter model

From the model fuction (8) we define six paramters and seven parameters models .

1. By taking $r=p$ in the model function (8), we get a model with six parameters and consider it as Model-A.

$$
d(t)=\left[\frac{s}{1+k\left[\frac{1+p}{\exp (q t+v)+p}\right]^{n}}\right], t \geq 0, d(0) \neq 0,
$$

2. By taking $r=p$ in the model function (8) and adding a parameter $m$, we get a model with seven parameters and consider it as Model-B.

$$
d(t)=m+\left[\frac{s}{1+k\left[\frac{1+p}{\exp (q t+v)+p}\right]^{n}}\right], t \geq 0, d(0) \neq 0 .
$$

3. The model function $\mathrm{d}(\mathrm{t})$ defined in $(8)$ is regarded as Model-C.

\subsection{Least squares parameter estimates}

To illustrate the applicability of the models, a data set from the USA is considered. Since this is a health-related problem, there is $95 \%$ confidence that results will be obtained using three models. Using the method of least squares, the model parameters in the functions (9), (10) and (8) are estimated with respect to the US data set (Table $1)$. and the plot of the data set is given in Figure 3

1. For the model function (9), $\mathrm{n}=1, \mathrm{p}=5.9771120 \mathrm{q}=0.17741590, \mathrm{k}=400.01300$, $\mathrm{v}=0.00092071428570$, and $\mathrm{s}=54900.0 .($ Model-A)

2. For the miodel function (10), $\mathrm{n}=1, \mathrm{p}=7.322220, \mathrm{q}=0.177940, \mathrm{k}=342.01860, \mathrm{~s}$ $=54800.0, \mathrm{v}=0.00123780$, and $\mathrm{m}=0.498040$. (Model-B)

3. For the model function (8), $\mathrm{n}=1, \mathrm{p}=-11.97474770, \mathrm{q}=0.15356040, \mathrm{k}=338.996880$, $\mathrm{s}=62100.0, \mathrm{v}=0.00064450$, and $\mathrm{m}=2.65862210$. $($ Model $-\mathrm{C})$ 


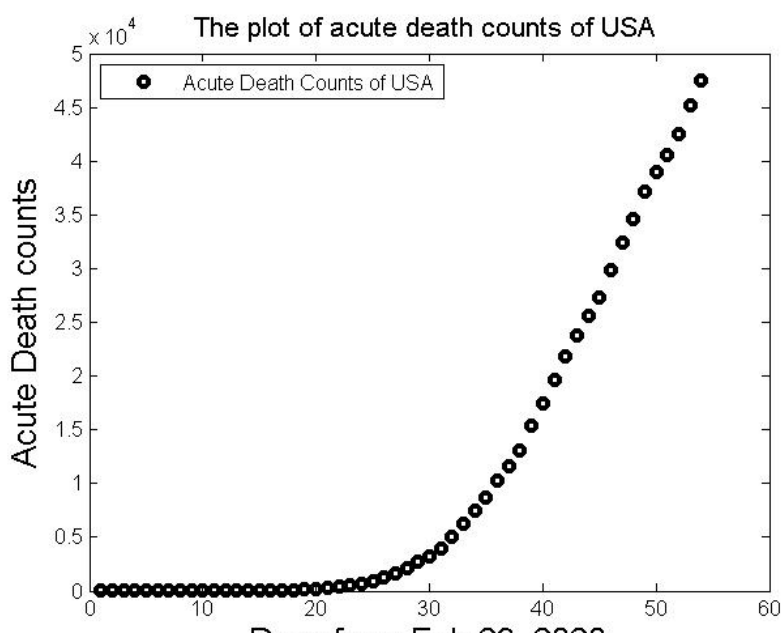

Days from Feb 29, 2020

Fig. 3 The plot of the Data set of mass acute deaths in USA due to COVID-19 virus .

\subsection{Comparison of the three proposed models}

The data sample size is 54 . We adapt the US data set to compare with the model functions (9), (10) and (8) with regard to the errors performed by the measurements SSE, MSE, SD, SSR, $R^{2}, \mathrm{R}, \mathrm{SST}, \gamma^{2}, \gamma, \mathrm{AIC}, \mathrm{BIC}, \mathrm{PIC}, \mathrm{PC}, \mathrm{PRR}$ and PP [46]. The best and optimal model function is selected based on the smallest error.

Model-A is a 6-parameter model, and Model-B and Model-C are 7-parameter models. It should be noted that to model the uncertainty of COVID-19 virus large number of infection and the mass acute deaths, more parameters need to be introduced into existing models. Thus, the comparison between two models with 7 parameters gives a better result. Model-A is obtained from Model-I by [75,89], Model-B is obtained from Model-II by [75,89] and Model-C is obtained from Model-III by [75, 89]. It can be seen from Table 2 and Table 4 that the Model-I error was decreased by the introduction of Model-A, the Model-II error was decreased by the introduction of Model B, and the Model-III error was decreased by the introduction of ModelC. Therefore, Model-A is better than Model-I, Model-B is better than Model-II, and Model-C is better than Model-III.

SSE, MSE, AIC, PIC, PC, BIC, PP, and PRR refer to the sum of the quadratic error, the quadratic mean error, the Akaike information criterion, the Pham information criterion, the Pham criterion, the Bayesian information criterion, the predictive power or the risk of a predictive relationship. And these are defined in [75]. The other key figures are defined in [46]. par When comparing the three models, errors based on SSE, AIC, BIC, PIC, and PC select model C as the best model. However, by only comparing models with 6 parameters, Errors due to SSE, MSE, SD, AIC, BIC, PIC, and PC select Model C as the best model. Overall, the PC error compared to all measurements is smaller than the other measurements and Model-C is the best model in Table 2. 
Table 2 Comparison of error criteria of the proposed three Models

\begin{tabular}{llll}
\hline $\begin{array}{l}\text { Error } \\
\text { criteria }\end{array}$ & $\begin{array}{l}\text { Model-A } \\
\text { 6-parameters }\end{array}$ & $\begin{array}{l}\text { Model-B } \\
\text { 7-parameters }\end{array}$ & $\begin{array}{l}\text { Model-C } \\
\text { 7-parameters }\end{array}$ \\
\hline Mean & 880.0000000000000 & 880.00000000000000 & 880.00000000000000 \\
SSE & 16281903.578350032 & 16767627.658993214 & 16178764.313820602 \\
MSE & 339206.32454895898 & 356758.03529772797 & 344229.02795362985 \\
SD & 582.41422076470542 & 597.29225283585254 & 586.71034416791213 \\
SSR & 16815203308.809811 & 16810094792.523708 & 16919054002.859499 \\
R $^{2}$ & 0.99903171533051 & 0.99900301379168 & 0.99904375479202 \\
R & 0.99951574041158 & 0.99950113871249 & 0.99952176304071 \\
SST & 16835173565.000000 & 16835173565.00000 & 16835173565.000000 \\
$\gamma^{2}$ & 0.99881377782575 & 0.99851033478334 & 1.00498245162342 \\
$\gamma$ & 0.99940671291809 & 0.99925488979706 & 1.00248813041523 \\
AIC & 699.65564672346841 & 704.37990516953209 & 358.22468733146690 \\
BIC & 711.58955100285402 & 718.30279349548198 & 372.14757565741684 \\
PIC & 16281910.203350032 & 16767635.552610235 & 16178772.207437623 \\
PC & 309.42293902134389 & 305.07406744653593 & 304.23392917051632 \\
PRR & 17.66249945494801 & 17.93586058669838 & 53.05513444830775 \\
PP & 42196.489974328841 & 57008.663891851356 & 605121.51493479579 \\
\hline
\end{tabular}

Table 3 Comparison of Predicted Data using the proposed three Models.

\begin{tabular}{llllll}
\hline Date & Models & $\begin{array}{l}\text { Mass Acute Death } \\
\text { count }\end{array}$ & $\begin{array}{l}\text { Accuracy of } \\
\text { confidence } \\
\text { in } \%\end{array}$ & $\begin{array}{l}\text { Error of } \\
\text { significance } \\
\text { in \% }\end{array}$ & $\begin{array}{l}\text { Best } \\
\text { model }\end{array}$ \\
\hline & Real data & 47,520 & & & \\
April 22, & Model-A & 46024.05114032712300 & 96.85195947 & 3.14804053 & \\
2929 & Model-B & 46002.93871353800900 & 96.80753096 & 3.192469045 & Model-C \\
& Model-C & 47341.11750397019900 & 99.62356376 & 0.376436237 & \\
\hline prediction & Real data & 49,845 & & & \\
April 23, & Model-A & 47265.92345853027700 & 94.8258069 & 5.174193099 & \\
2929 & Model-B & 47238.60868548924200 & 94.77100949 & 5.228990511 & Model-C \\
& Model-C & 49003.25389489919300 & 98.31127273 & 1.688727275 & \\
\hline
\end{tabular}

3.4 Comparison of Predicted Data of the USA using proposed three models

In the U.S. dataset, the mass acute death count as of April 22, 2020, is 47,520. Data of $55^{\text {th }}$ day as of April 23, 2020, are extrapolated from the data set using three proposed models and compared to the actual data. They are listed in Table 3. For the best Model-C on April 22, 2020, we observe that $99.62356376 \%$ accuracy with the fitted data. And on April 23, 2020, the predicted data is observed to have an accuracy of $98.31127273 \%$. Thus, the significance error based on the data adjusted to April 22, 2020 is $0.376436237 \%<0.4 \%$ and the significance error based on the forecast of April 23, 2020 is $1.688727275 \%<2 \%$. Model-C is the best choice, and is a significant fit based on US mass acute death data. 
Table 4 Comparison of error criteria with the three Models of Phem.[75, 89]

\begin{tabular}{llll}
\hline $\begin{array}{l}\text { Error } \\
\text { criteria }\end{array}$ & $\begin{array}{l}\text { Model-I } \\
\text { 4-parameters- }\end{array}$ & $\begin{array}{l}\text { Model-II } \\
\text { 5-parameters }\end{array}$ & $\begin{array}{l}\text { Model-III } \\
\text { 5-parameters }\end{array}$ \\
\hline Mean & 880.000000000000 & 880.0000000000000 & 880.0000000000000 \\
SSE & 16284073.541271631 & 16769679.71861113 & 16181430.310243983 \\
MSE & 325681.47082543263 & 342238.36160430877 & 330233.27163763228 \\
SD & 570.68508901620396 & 585.01142006315467 & 574.65926568500765 \\
SSR & 16826082754.9035910 & 16824663464.164364 & 16928442136.545977 \\
R $^{2}$ & 0.99903221244193 & 0.99900326804430 & 0.99904412761790 \\
R & 0.99951598908768 & 0.99950150977590 & 0.99952194954283 \\
SST & 16835173565.0000000 & 16835173565.00000 & 16835173565.00000 \\
$\gamma^{2}$ & 0.99946001090744 & 0.99937570582239 & 1.00554010157281 \\
$\gamma$ & 0.99972996899535 & 0.99968780417808 & 1.00276622478662 \\
AIC & 693.45845536404499 & 698.13618779981368 & 353.10397332164740 \\
BIC & 701.41439155030207 & 708.08110803263503 & 363.04889355446880 \\
PIC & 16284077.7812716310 & 16769685.126774395 & 16181435.718407247 \\
PC & 319.65785145495096 & 315.23758848551461 & 314.36273833116815 \\
PRR & 17.66803741099453 & 17.94289695446730 & 54.37948833700809 \\
PP & 42211.2647253312900 & 57031.199814323576 & 605026.79799101199 \\
\hline
\end{tabular}

The discussion above is all about predicting the highest number of the mass acute deaths in the US from COVID-19 disease in the coming days. The data analysis of this section motivates to do the work in this article to predict the lifespan of the COVID-19 coronavirus using mathematical models.

\subsection{Comparison of three Models of Phem.[75,89]}

In Model-A, Model-B, and Model-C of this article, if we take $\mathrm{n}-1$ and $\mathrm{v}=0$, we get Model-I, Model-II, and Model-III respectively, the models of Phem. [75]. Model-I is a 4-parameter model, and the Model-II and the Model-III are 5-parameter models. It should be noted that parameters are introduced into existing models to model the uncertainty of COVID-19 viral large number of infections and mass acute deaths. Thus, the comparison between two models with 5 parameters gives a better result.

When comparing the three models, errors based on SSE, AIC, BIC, PIC, and PC select Model-III as the best model. However, by only comparing models with 5 parameters, Errors due to SSE, MSE, SD, AIC, BIC, PIC, and PC select Model-III as the best model. Overall, the PC error compared to all measurements is smaller than the other measurements and Model-III is the best model in Table 4.

\subsection{Comparison of Predicted Data of USA using the three Models of Phem.[75]}

In the US dataset, the data as of April 22, 2020, is 47,520 and is consistent with the data using Model-I, Model-II, and Model-III values received on April 22, 2020. Data of $55^{\text {th }}$ day as of April 23, 2020, are extrapolated from the data set using these three models and compared to the actual data. They are listed in Table 5. For Best Model-III on April 22, 2020, and accuracy of $99.63886681 \%$ is observed in the fitted 
Table 5 Comparison of Predicted Data of the three Models of Phem.[75, 89]

\begin{tabular}{llllll}
\hline Date & Models & $\begin{array}{l}\text { Mass Acute Death } \\
\text { count }\end{array}$ & $\begin{array}{l}\text { Accuracy of } \\
\text { confidence } \\
\text { in \% }\end{array}$ & $\begin{array}{l}\text { Error of } \\
\text { significance } \\
\text { in \% }\end{array}$ & $\begin{array}{l}\text { Best } \\
\text { model }\end{array}$ \\
\hline & Real data & 47,520 & & & \\
April 22, & Model-I [75] & 46030.897151574245 & 96.86636606 & 3.133633944 & \\
2929 & Model-II[75] & 46012.071788207242 & 96.82675036 & 3.173249621 & Model III \\
& Model-III [75] & 47348.389514346782 & 99.63886681 & 0.361133186 & \\
\hline prediction & Real data & 49,845 & & & \\
April 23, & Model-I[75] & 47271.970775468915 & 94.83793915 & 5.162060849 & \\
2929 & Model-II[75] & 47246.670252151780 & 94.78718076 & 5.21281924 & Model III \\
& Model-III[75] & 49009.930525869793 & 98.32466751 & 1.67533246 & \\
\hline
\end{tabular}

data. And on April 23, 2020, the predicted data is observed to have an accuracy of $98.32466751 \%$. Thus, the materiality error based on the data adjusted to April 22, 2020 , is $0.361133186 \%<0.4 \%$ and the materiality error based on the forecast of April 23, $20201.67533246 \%<2 \%$. Model-III is the best choice that fits the data significantly based on death dates in the United States.

The next sections explain three models for the life span of the coronavirus COVID19 in the United States.

\section{A dependent mathematical Model-A with 6 parameters and results}

These sections discuss a 6-parameter dependent mathematical model, Model-A, for the lifetime of the COVID-19 coronavirus in the United States. That is, this section calculates the lifespan of the COVID-19 coronavirus (the time without mass acute death from COVID-19) in the US using a mathematical model to better protect people and the environment and to speed up the general pandemic preparedness. Everyone is afraid and not sure of their death. Everyone in the world is afraid of mass acute deaths. From now on, it is the main responsibility of researchers to give hope to living and active people. The main purpose of this article is to predict the time of no mass acute death due to COVID-19. Or when we can live in peace without fear of COVID-19? This article answer this question using three mathematical models. This prediction using a mathematical model is completely new.

The growth function of mass acute death can be defined by the logistic function

$$
d_{1}(t)=\left[\frac{s}{1+k\left[\frac{1+p}{\exp (q t+v)+p}\right]^{n}}\right], t \geq 0,
$$

where $\mathrm{p}=5.977112, \mathrm{q}=0.1774159, \mathrm{k}=400.013, \mathrm{~s}=54900, \mathrm{v}=0.0009207142857$, and $n=1$. In Figure 4, the numbers of mass acute deaths in the United States are from February 29, 2020 through April 22, 2020 that correspond to the function (11) is plotted. The function (11) fulfills the hypotheses (6) and (7). This is a six parameter model that takes into account the uncertainty of the COVID-19 coronavirus in the population. A six-parameter model is used to improve the goodness of fit of the adaptation to the data set. 


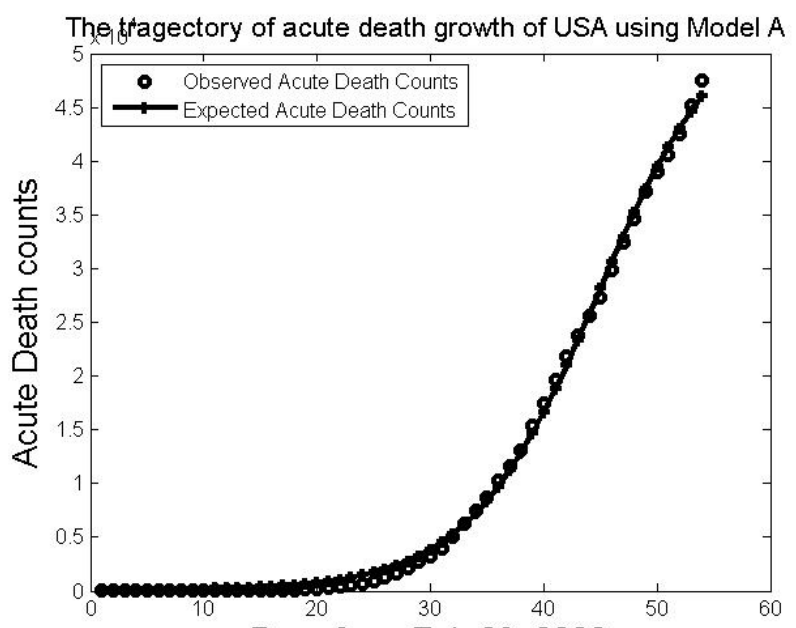

Days from Feb 29, 2020

Fig. 4 The mass acute death growth trajectory of USA using the mathematical model, Model-A

The probability density function of no mass acute death due to COVID-19 is given by

$$
f_{1}(t)=\left[\frac{\left(d_{1}\right)^{\prime}(t)}{T}\right] \exp \left(-\left[\frac{d_{1}(t)}{T}\right]\right), t \geq 0, t \in(0, T), T>0
$$

where

$$
\left(d_{1}\right)^{\prime}(t)=\left[\frac{(n) q k(1+p)^{n}}{s}\right]\left[\frac{\exp (q t+v)}{(\exp (q t+v)+q)^{1+n}}\right]\left[d_{1}(t)\right]^{2}, t \geq 0
$$

4.1 Prediction of the time of no mass acute death due to COVID-19 using the total number of mass active deaths $\mathrm{s}$

The probability density function $f_{1}(t)$ reaches the value zero when $t$ approaches a larger value. For which value of $t$ does the function $f_{1}(t)=0$ apply, which we have to calculate in this section. If $t$ takes a larger value, the function $d_{1}(t)$ approaches $\mathrm{s}$. Since $\mathrm{t}$ takes a larger value, the function $\exp \left(-\left[\frac{d_{1}(t)}{T}\right]\right)$ reaches zero and therefore $f_{1}(t)$ is close to zero. From then on, when the deathly COVID-19 disease stops killing the people in the United States, or when there will be no mass acute death or lifetime can be be determined from the relationship.

$$
\text { lifetime }=\frac{s}{228} .
$$

We have the time without the mass acute death or lifetime will be 240.789473684210 $\approx 241$ days. From February 29, 2020, there are 241 days. In other words, there will be no mass acute deaths from COVID-19 in the US after November 1, 2020. This is not the case compared to live updates from the WHO. Again we examine this model in the next subsection. 
4.2 Prediction of the time of no mass acute death due to COVID-19 using the acute death rate $\mathrm{q}$

The probability density function $f_{1}(t)$ reaches the value zero when $t$ approaches a larger value. In this section we have to calculate for which value of the function $f_{1}(t)=0$ reaches zero. Again, $\left(d_{1}\right)^{\prime}(t)$ can be rewritten as follows:

$$
\left(d_{1}\right)^{\prime}(t)=\left[\frac{(n) q k(1+p)^{n}}{s}\right]\left[\frac{\exp (-(q t+v))}{(1+q \cdot \exp (-(q t+v)))^{1+n}}\right]\left[d_{1}(t)\right]^{2}, t \geq 0,
$$

As t becomes larger, the function $\exp (-(q t+v))$ approaches zero and therefore $f_{1}(t)$ approaches zero. From there, if COVID-19 mass acute death disease stops killing people in the United States or when there will be no mass acute death or lifetime can calculated from the relationship.

$$
\text { lifetime }=\left(\frac{228}{q(n)}\right)-\left(\frac{v}{q}\right)
$$

We have calculated that as of February 29, 2020, the time without mass acute death will be $1285.12112338457680 \approx 1286$. That is, after 3 years, 6 months, and 24 days from February 29, 2020 (from September 2023). There will be nomass acute deaths from COVID-19 in the US worldwide if everyone follows WHO guidelines and advice from health workers. Therefore, using the mathematical model Model-A, the US COVID-19 coronavirus lifespan is calculated to be 1,286 days from February 29, 2020 .

\section{A mathematical Model-B that depends on 7 parameters and results}

This section, a mathematical model that depends on 7 parameters, discusses ModelB for the lifespan of the COVID-19 coronavirus in the United States. That is, this section calculates the lifespan of the COVID-19 coronavirus (the time without mass acute death from COVID-19) in the US using a mathematical model to better protect people and the environment and to speed up the general pandemic preparedness. This prediction using a mathematical model is completely new.

The growth function of the mass acute death can be defined by the logistic function

$$
d_{2}(t)=m+\left[\frac{s}{1+k\left[\frac{1+p}{\exp (q t+v)+p}\right]^{n}}\right], t \geq 0,
$$

where $\mathrm{p}=7.32222, \mathrm{q}=0.17794, \mathrm{k}=342.0186, \mathrm{~s}=54800, \mathrm{~m}=0.49804, \mathrm{v}=0.0012378$, and $n=1$. Figure 5 shows the mass acute deaths in the United States drawn from February 29th to April 22nd, 2020, which corresponds to the function (17). It is a seven parameter model that takes into account the uncertainty of the COVID-19 coronavirus in the population. The seven-parameter model is used to improve the goodness of fit of the adaptation to the data set.

The probability density function of no mass acute death due to COVID-19 is given by

$$
f_{2}(t)=\left[\frac{\left(d_{2}\right)^{\prime}(t)}{T}\right] \exp \left(-\left[\frac{d_{2}(t)}{T}\right]\right), t \geq 0, t \in(0, T), T>0
$$




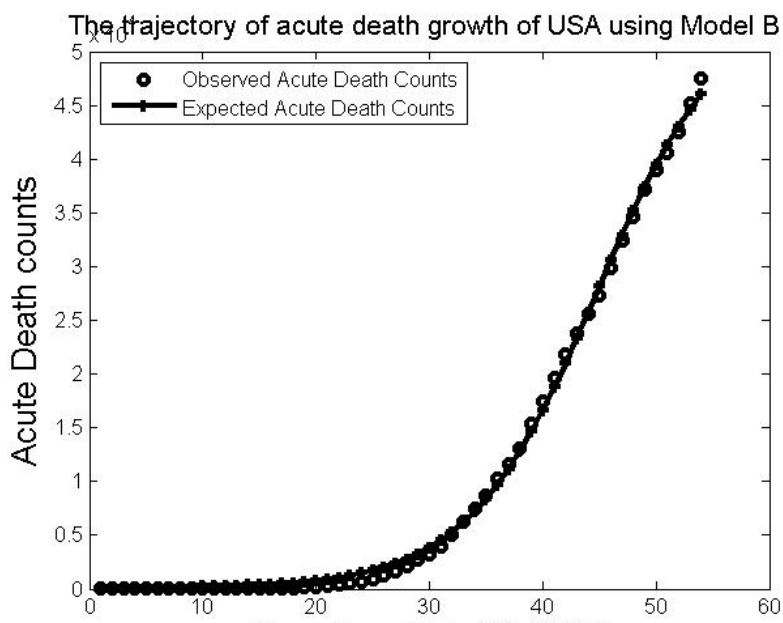

Days from Feb 29, 2020

Fig. 5 The mass acute death growth trajectory of USA using the mathematical model, Model-B

where

$$
\left(d_{2}\right)^{\prime}(t)=\left[\frac{(n) q k(1+p)^{n}}{s}\right]\left[\frac{\exp (q t+v)}{(\exp (q t+v)+q)^{2-n}}\right]\left[d_{2}(t)-m\right]^{2}, t \geq 0 .
$$

5.1 Prediction of the time of no mass acute death due to COVID-19 using the total number of mass acute deaths $\mathrm{s}$

The probability density function $f_{2}(t)$ reaches the value zero when $t$ approaches a larger value. For which value of $t$ does the function $f_{2}(t)=0$ apply, which we have to calculate in this section. When $t$ takes on a larger value, the function $d_{2}(t)$ approaches $\mathrm{m}+\mathrm{s}$. When $\mathrm{t}$ takes on a larger value, the function $\exp \left(-\left[\frac{d_{2}(t)}{T}\right]\right)$ approaches zero and therefore $f_{2}(t) \mathrm{s}$ approaches zero. From then on, when the deathly COVID-19 disease stops killing the people in the United States, or when there will be no mass acute death or lifetime can be determined from the relationship.

$$
\text { lifetime }=\frac{m+s}{228}
$$

We have the time without mass acute deaths or lifetime will be about 240.35306157894738 $\approx 241$ days. From February 29, 2020, there are 241 days. In other words, there will be nomass acute deaths from COVID-19 in the US after November 1, 2020, provided everyone follows WHO guidelines and advice from health workers. This is not the case compared to live updates from the WHO. Again we examine this model in the next subsection. 
5.2 Prediction of the time of no mass acute death due to COVID-19 using the acute mass death rate $\mathrm{q}$

The probability density function $f_{2}(t)$ reaches the value zero when $t$ approaches a larger value. In this section we have to calculate for which value of $t$ the function $f_{2}(t)=0$ reaches zero. Again, $\left(d_{2}\right)^{\prime}(t)$ can be rewritten as follows:

$$
\left(d_{2}\right)^{\prime}(t)=\left[\frac{(n) q k(1+p)^{n}}{s}\right]\left[\frac{\exp (-(q t+v))}{\left.(1+q \cdot \exp (-(q t+v)))^{1+n}\right]\left[d_{2}(t)-m\right]^{2}, t \geq 0 .(21)}\right.
$$

When $t$ takes a larger value, the function $\exp (-(q t+v))$ approaches zero and therefore $f_{2}(t)$ approaches zero. From there, the relationship can determine when themass acute death-causing COVID-19 disease will stop killing the people of the United States or when there will be no mass acute deaths or lifetime can be calculated from the relationship

$$
\text { lifetime }=\left(\frac{228}{q(n)}\right)-\left(\frac{v}{q}\right) .
$$

We have calculated that as of February 29, 2020, the time without mass acute death will be $1281.3377719194848400 \approx$. That is, after 3 years, 6 months, and 22 days from February 29, 2020 (as of September 2023), there will be no mass acute deaths in the US from COVID-19 shipping worldwide if all of WHO guidelines and follow the advice of health workers. Therefore, using the mathematical model Model B, the US COVID-19 coronavirus lifespan is calculated to be 1,282 days from February 29, 2020

\section{A dependent mathematical Model-C with 7 parameters and results}

These sections, a mathematical model that depends on 7 parameters, discuss Model$\mathrm{C}$ for the lifespan of the COVID-19 coronavirus in the United States. That is, this section calculates the life span of the COVID-19 coronavirus (the time without mass acute death due to COVID-19) in the United States using the best mathematical model to better protect people and pandemic preparedness. This prediction using a mathematical model is completely new.

The growth function of the mass acute death can be defined by the logistic function

$$
d_{3}(t)=\left[\frac{s}{1+k\left[\frac{1+p}{\exp (q t+v)+r}\right]^{n}}\right], t \geq 0,
$$

where $\mathrm{r}=-11.9747477, \mathrm{q}=0.1535604, \mathrm{k}=338.99688, \mathrm{~s}=62100, \mathrm{p}=2.6586221$, $\mathrm{v}=0.0006445$ and $\mathrm{n}=1$. In Figure 6 the number of mass acute deaths is in the United States from February 29 to April 22, 2020 is plotted, which corresponds to the function (23). The function (23) fulfills the hypotheses (6) and (7). It is a seven parameter model that takes into account the uncertainty of the COVID-19 coronavirus in the population. A seven-parameter model is used to improve the quality of the adaptation to the data set. 


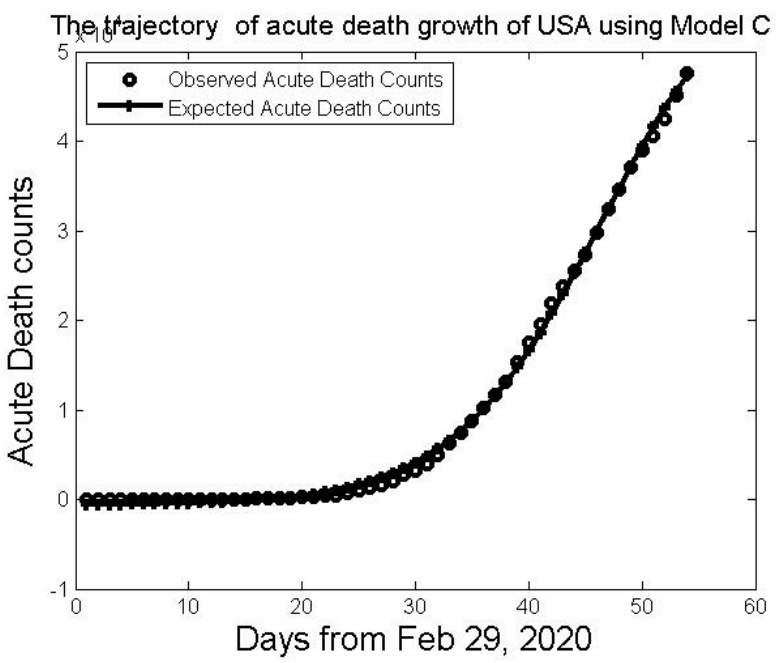

Fig. 6 The mass acute death growth trajectory of USA using the mathematical model, Model-C

The probability density function of no mass acute death due to COVID-19 is given by

$$
f_{3}(t)=\left[\frac{\left(d_{3}\right)^{\prime}(t)}{T}\right] \exp \left(-\left[\frac{d_{3}(t)}{T}\right]\right), t \geq 0, t \in(0, T), T>0
$$

where

$$
\left(d_{3}\right)^{\prime}(t)=\left[\frac{(n) q k(1+p)^{n}}{s}\right]\left[\frac{\exp (q t+v)}{(\exp (q t+v)+q)^{1+n}}\right]\left[d_{3}(t)\right]^{2}, t \geq 0
$$

6.1 Prediction of the time of no mass acute death due to COVID-19 using the total number of death $\mathrm{s}$

The probability density function $f_{3}(t)$ reaches the value zero when $t$ approaches a larger value. In this section we have to calculate for which value of the function $f_{3}(t)=0$ reaches zero. When $t$ takes on a larger value, the function $d_{3}(t)$ approaches $\mathrm{s}$. When $\mathrm{t}$ takes on a larger value, the function $\exp \left(-\left[\frac{d_{3}(t)}{T}\right]\right)$ approaches zero and therefore $f_{3}(t)$ approaches zero. From there, the relationship can determine when the mass acute death-causing COVID-19 disease will stop killing the people of the United States or when there will be no mass acute deaths or the lifetime is calculated from the relationship

$$
\text { lifetime }=\frac{s}{228} .
$$

We have the time without mass acute deaths will be $274.77876106194691 \approx 275$ days. From the first day of acute death, February 29, 2020, there are 272 days. In other words, there will be no mass acute deaths from COVID-19 in the US after December 1, 2020, provided everyone follows WHO guidelines and advice from health workers. This is not the case compared to live updates from the WHO. Again we examine this model in the next subsection 
6.2 Prediction of the time of no mass acute death due to COVID-19 using the death rate $\mathrm{q}$

The probability density function $f_{3}(t)$ reaches the value zero when $t$ approaches a larger value. In this section we have to calculate for which value of the function $f_{3}(t)=0$ reaches zero. Again, $\left(d_{3}\right)^{\prime}(t)$ can be rewritten as follows:

$$
\left(d_{3}\right)^{\prime}(t)=\left[\frac{(n) q k(1+p)^{n}}{s}\right]\left[\frac{\exp (-(q t+v))}{(1+q \cdot \exp (-(q t+v)))^{1+n}}\right]\left[d_{3}(t)\right]^{2}, t \geq 0 .
$$

As $t$ takes a larger value, the function $\exp (-q t)$ approaches zero and therefore $f_{3}(t)$ approaches zero. From there, when the COVID-19 mass acute death disease stops killing people in the United States, or if when there will be no mass acute deaths or lifetime can be determined from the relationship.

$$
\text { lifetime }=\left(\frac{228}{q(n)}\right)-\left(\frac{v}{q}\right) .
$$

We have no mass acute -death time of $1484.76198616309920 \approx 1485$ as of February 29, 2020. That is 4 years, 1 month, and 15 days as of February 29, 2020 ( from April 2024). There will be no mass acute deaths from COVID-19 in the US worldwide if everyone follows WHO guidelines and advice from health workers. Therefore, using the Model-C mathematical model, the US COVID-19 coronavirus lifespan is calculated to be 1,485 days from the first day of acute death, February 29, 2020.

\section{Comparison of the proposed models and the Models of Phem.[75,89]}

This section compares the proposed models for a lifetime of the COVID-19 virus in the US and determines that Model-C is the model that best fits the US dataset. And in this section the proposed models and the models of Phem. [75] are compared based on the error criteria and it is determined that Model-C is the most suitable model for the US data set.

\subsection{Comparison of proposed three Models for Lifetime of COVID-19 virus}

Three proposed models are compared in Table 6. We assume the maximum value got from Model-C, calculated from Table 6 to be 274.77876106194691 days, based on the total number of mass acute deaths at time $t$. Using the maximum value, three proposed models predict that after 274.77876106194691 days from the first day of acute death from February 29, 2020 the mass acute death will not occur and serious cases will occur compared to live updates from the WHO. U.S. the mass acute deaths from COVID-19 continue even after December 2020.

The value from Model-C, calculated from these three proposed models at 1484.76198616309920 days based on the mass acute death rate. Therefore, we cannot expect any mass acute deaths from COVID-19 worldwide in the US after January 2025 if everyone follows WHO guidelines and advice from health workers. The best model among 
Table 6 Comparison of proposed Models for Lifetime of COVID-19 virus in the USA.

\begin{tabular}{lll}
\hline Models & Metrics & \\
\hline & Total number of acute death occurs at time t & The acute death rate \\
\hline Model-A & 240.78947368421052 & 1285.12112338457680 \\
Model-B & 240.35306157894738 & 1281.33774193548400 \\
Model-C & 274.77876106194691 & 1484.76198616309920 \\
\hline
\end{tabular}

the 6 and 7 parameter models is Model-C (the 7 parameter model) which predicts the life span of the COVID-19 coronavirus in the US on February 29, 2020, at 1484.76198616309920 days.

7.2 Comparison of error criteria with the proposed models and the Models of Phem. $[75,89]$

By comparing the error criteria regarding the errors caused by the measurements SSE, MSE, SD, SSR, $R^{2}, \mathrm{R}, \mathrm{SST}, \gamma^{2}, \gamma$, AIC, BIC, were made. PIC, PC, PRR, and PP [46] with the proposed models and the models of Phem. [75] from Table 7 (Table 2 and Table 4), the proposed model, Model-C, best fits the model to the US dataset. We observe that Model-C is better at

1. The sum of the squares of the SSE errors is minimized by comparing the 6 models.

2. The AIC value was minimized by comparing the 6 models.

3. The BIC value was minimized by comparing the 6 models.

4. The PIC value was minimized by comparing the 6 models.

5. The PC value was minimized by comparing the 6 models.

6. The values of $\gamma^{2}$ and $\gamma$ are approximately equal to 1 , which implies the use of the Model-C model and offers $100 \%$ fitness with the specified data set.

7. The values of $R^{2}$ and $\mathrm{R}$ are approximately equal to 0.99904375479202 , and 0.99952176304071 involves the use of the Model-C, which offers a fitness of $99.95 \%$ with the specified data set.

7.3 Comparison of proposed models and Models of Phem.[75,89] for Lifetime of COVID-19 virus.

Three proposed and existing models are compared in Table 8. We take the maximum value from Model-C, calculated from Table 8 at 274.77876106194691 days based on the total number of the mass acute deaths at time t. No mass acute death is predicted using six models after 274.77876106194691 days from the first day of acute death from February 29, 2020 and the mass acute death compared to WHO's live updates in the US even after December 2020 due to COVID-19.

We take the maximum value from Model-C, calculated from these six models (see Table 8). 1484.76198616309920 days based on the acute death rate. Therefore, 
Table 7 Comparison of error criteria with the proposed models and the Models of Phem.[75,89]

\begin{tabular}{lllllll}
\hline Error & Model-I & Model-II & Model-III & Model-A & Model-B & Model-C \\
\hline Mean & 880.0000000000000 & 880.00000000000000 & 880.00000000000000 & 880.00000000000000 & 880.000000000000000 & 880.0000000000000000 \\
SSE & 16284073.5412716310 & 16769679.718611130 & 16181430.310243983 & 6284073.5412716310 & 16769679.718611130 & 16181430.310243983 \\
MSE & 325681.470825432630 & 342238.36160430877 & 330233.27163763228 & 325681.470825432630 & 342238.36160430877 & 330233.27163763228 \\
SD & 570.68508901620396 & 585.01142006315467 & 574.65926568500765 & 570.68508901620396 & 585.01142006315467 & 574.65926568500765 \\
SSR & 16826082754.9035910 & 16824663464.164364 & 16928442136.545977 & 16826082754.9035910 & 16824663464.164364 & 16928442136.545977 \\
R $^{2}$ & 0.99903221244193 & 0.99900326804430 & 0.99904412761790 & 0.99903221244193 & 0.99900326804430 & 0.99904412761790 \\
R & 0.99951598908768 & 0.99950150977590 & 0.99952194954283 & 0.99951598908768 & 0.99950150977590 & 0.99952194954283 \\
SST & 16835173565.0000000 & 16835173565.00000 & 16835173565.00000 & 16835173565.0000000 & 16835173565.00000 & 16835173565.00000 \\
$\gamma^{2}$ & 0.99946001090744 & 0.99937570582239 & 1.00554010157281 & 0.99946001090744 & 0.99937570582239 & 1.00554010157281 \\
$\gamma$ & 0.99972996899535 & 0.99968780417808 & 1.00276622478662 & 0.99972996899535 & 0.99968780417808 & 1.00276622478662 \\
AIC & 693.45845536404499 & 698.13618779981368 & 353.10397332164740 & 693.45845536404499 & 698.13618779981368 & 353.10397332164740 \\
BIC & 701.41439155030207 & 708.08110803263503 & 363.04889355446880 & 701.41439155030207 & 708.08110803263503 & 363.04889355446880 \\
PIC & 16284077.7812716310 & 16769685.126774395 & 16181435.718407247 & 16284077.7812716310 & 16769685.126774395 & 16181435.718407247 \\
PC & 319.65785145495096 & 315.23758848551461 & 314.36273833116815 & 319.65785145495096 & 315.23758848551461 & 314.36273833116815 \\
PRR & 17.66803741099453 & 17.94289695446730 & 54.37948833700809 & 17.66803741099453 & 17.94289695446730 & 54.37948833700809 \\
PP & 42211.2647253312900 & 57031.199814323576 & 605026.79799101199 & 42211.2647253312900 & 57031.199814323576 & 605026.79799101199 \\
\hline
\end{tabular}


Table 8 Comparison of proposed models and Models of Phem.[75,89] for Lifetime of COVID-19 virus in the USA.

\begin{tabular}{lll}
\hline Models & Metrics & \\
\hline & Total number of acute death occurs at time t & The acute death rate \\
\hline Model-I [75] & 240.78947368431052 & 1285.1159338030020 \\
Model-II [75] & 240.35306157894738 & 1281.3307856580871 \\
Model-III[75] & 272.37876106194692 & 1464.7577891175067 \\
Model-A & 240.78947368421052 & 1285.12112338457680 \\
Model-B & 240.35306157894738 & 1281.33774193548400 \\
Model-C & 274.77876106194691 & 1484.76198616309920 \\
\hline
\end{tabular}

we cannot expect any mass acute deaths from COVID-19 worldwide in the US after January 2025 if everyone follows WHO guidelines and advice from health workers. The best model among the 4-parameter, 5-parameter, 6-parameter, and 7-parameter models is Model-C (the 7-parameter model) which is the lifetime of the COVID-19 coronavirus in the United States at 1484.76198616309920 days forecast from the first day of acute death, February 29, 2020.

7.4 Comparison of Predicted Data using proposed models and Models of Phem[75, 89]

Tables 3 and 5 show that on April 22, 2020, the number of mass acute deaths using Model C is 47341.11750397019900 . The confidence level is $99.62356376 \%$ with a significance error of $0.376436237 \%$. Tables 3 and 5 again show that as of April 23, 2020, the number of mass acute deaths predicted using the Model-C is 49003.25389489919300. The confidence level is $98.31127273 \%$ with a significance error of $1.688727275 \%$.

\section{Prediction of total mass acute death counts based on the data set of USA}

In this section, data analysis is performed to predict that the data set will miss certain counts during data collection and it is identified. Based on the ratio of the standard deviation to the mean of mass acute deaths, the total number of mass acute deaths over 54 days is estimated at 62,969 . The standard deviation around the line of regression shows that a large number is missing from the data set when collecting data from the United States. Using the coefficient of determination, it is predicted that the Model-C will provide $100 \%$ fitness with the given data set and only $0.0 \%$ variation. All three models are eligible for the US dataset, but Model-C is the best of the three. This section also predicts from Model-A, Model-B, and Model-C that the total mass acute deaths over 54 days will be 66,557, 67085, and 68,523, respectively. The best model is Model-C, the total mass acute deaths predicted for 54 days will be 68,523 . 
8.1 Prediction using the data set

This section uses mass acute death counts of U.S. censuses from February 2, 2020 through April 22, 2020. The number of mass acute deaths for 54 days is $s_{54}=47,520$. The average number of mass acute deaths per day is 880 . Therefore, the total number of mass acute deaths for 54 days is the product of the sample size and the average number of mass acute deaths is 47,520 .

The standard deviation of the number of mass acute deaths is 17656 . The ratio of the standard deviation to the average number of mass acute deaths per day is approximately 2.006.363636, a value greater than one. This indicates that a large number is missing from the data set when collecting data in the United States.

The minimum number of mass acute deaths during these 54 days would be 47,520 $+13,242=60,762$. The maximum number of mass acute deaths is 47,520 $+17,656$ $=65,176$. Therefore, the total expected count (PTDC) during and up to 54 days in the United States with a $95 \%$ confidence level is 62,969 . The confidence interval of PTDC is [60762, 65176]. This means

$$
60,762 \leq P T D C \leq 65,176 .
$$

The United States has 50 states and 94 counties. Given the minimum of 3 acute deaths per day from each district that are not counted, we get a total of 15,228 missed mass acute deaths for 54 days. So the total number of mass acute deaths will be 62,748 . Therefore, changes in the number of US records can be expected.

\subsection{Prediction using standard deviation around the line of regression}

With reference to the models Model-A, Model-B and Model-C, we analyze the data set. The standard deviation aroind the line of regression(SDALR) is the standard error of the estimate and is given by

$$
S D A L R=\sqrt{\frac{S S E}{n-k}} .
$$

Here, $\mathrm{n}$ and $\mathrm{k}$ stand for the size of the data set and the number of parameters in the model. We watch that

1. For the Model-A, SDALR $=582.4142206$,

2. For the Model-B, SDALR $=597.2922527$,

3. For the Model-C, SDALR $=586.7103441$.

This indicates that a large number is missing from the data set when collecting data in the United States.

\subsection{Prediction using the coefficient of determination}

In terms of Model-A, Model-B, and Model-C, we analyze the data set. We use the regression coefficient to measure the variations in the data set. The regression coefficient is the ratio of the sum of the regression squares to the total sum of the squares. 
And it is expressed as

$$
\gamma^{2}=\frac{S S R}{S S T}
$$

where SSR and SST represent the regression sum of the squares and the real sum of squares, respectively. It is observed that

1. For the Model-A, $\gamma^{2}=0.99881377782575$,

2. For the Model-B, $\gamma^{2}=0.99851033478334$,

3. For the Model-C, $\gamma^{2}=1.00498245162342$.

Using Model-A provides $99.88 \%$ fitness with the specified data set and only $0.12 \%$ variation. Using Model-B provides $99.85 \%$ fitness with the specified data set and only $0.15 \%$ variation. Using the Model-C offers $100 \%$ fitness with the specified data set and only $0.0 \%$ variation. All three models are eligible for the US dataset, but Model-C is the best of the three.

\subsection{Prediction using the models}

We want to predict missing data in the US dataset using Model-A, Model-B, and Model-C through data analysis.

Using 6-parameter Model-A, we have,

1. $\operatorname{sum}=s_{54}=46024.05114032712300$,

2. mean $=\bar{x}=958.8343988$,

3. total sum $=\mathrm{n} \cdot \bar{x}=51777.05753$,

4. maximum $=\mathrm{n} \cdot \bar{x}+17656=69433.057531$,

5. minimum $=s_{54}+17656=63680.05114$,

6. predicted value $=\mathrm{PTDC}=66556.55434$.

The 6-parameter Model-A predicts that from February 29, 2020 to April 22, 2020, the number of mass acute deaths due to the COVID-19 coronavirus in the US will be almost 66,557 with a confidence level of $95 \%$ and the interval of confidence is [ 63681, 69434]. This means,

$$
63681 \leq P T D C \leq 69434
$$

The expected number of mass acute deaths will be between 63,681 and 69,434

Using 7-parameter Model-B, we have,

1. $\operatorname{sum}=s_{54}=46002.93871353800900$,

2. mean $=\bar{x}=978.78593$,

3. total sum $=\mathrm{n} \cdot \bar{x}=52854.44022$,

4. maximum $=\mathrm{n} \cdot \bar{x}+17656=70510.44022$,

5. minimum $=s_{54}+17656=63658.93871$,

6. predicted value $=\mathrm{PTDC}=67084.68947$. 
Model B predicts the mass acute death counts from February 29, 2020 to April 22, 2020 due to the COVID-19 coronavirus in the US will be nearly 67,085 with a confidence level of $95 \%$ and a interval of confidence is $[63659,70511]$. This means,

$$
63659 \leq P T D C \leq 70511 .
$$

The expected number of mass acute deaths will be between 63,659 and 70,511.

Using 7-parameter Model-C, we have,

1. sum $=s_{54}=47341.11750397019900$,

2. mean $=\bar{x}=1007.257819$,

3. total sum $=\mathrm{n} \cdot \bar{x}=54391.92223$,

4. maximum $=\mathrm{n} \cdot \bar{x}+17656=72047.92223$,

5. minimum $=s_{54}+17656=64997.1475$,

6. predicted value $=\mathrm{PTDC}=68522.53487$

Model-C predicts that between February 29 and April 22, 2020, the number of mass acute deaths from the COVID-19 coronavirus in the United States will be nearly 68,523 with a confidence level of $95 \%$ and a confidence interval of [64998, 72048] becomes ]. This means,

$$
64998 \leq P T D C \leq 72048 .
$$

The expected number of mass acute deaths will be between 64,998 and 72,048.

Finally, from Model-A, Model-B, and Model-C, the total number of mass acute deaths over 54 days is predicted to be $66,557,67,085$, and 68,523 , respectively. The best model is Model-C, the total mass acute deaths predicted for 54 days will be 68,523

The borders of the United States are bordered by the Atlantic Ocean to the east, the Pacific Ocean to the west, Canada to the north, and Mexico and the Gulf of Mexico to the south. The radius of the geographic location on Earth is 3,881 miles from downtown Kansas. The total area 9,834 million $\mathrm{km}^{2}$ From sea level, Badwater in Death Valley is the lowest point at $86 \mathrm{~m}$ below sea level and Denali is the highest point at $6,194 \mathrm{~m}$ above sea level.

The COVID-19 virus is round or elliptical and often pleomorphic in shape and around 60 to $140 \mathrm{~nm}$ in diameter. Recent research has examined the survival of the COVID-19 virus on various surfaces and reports that the virus can remain viable in plastic and stainless steel for up to 72 hours, in copper for up to 4 hours, and cardboard boxes for up to 24 hours time. The virus COVID-19 mainly spreads through the air when people are close together. Allows an infected person to breathe, cough, sneeze, or speak, and enter another person through their mouth, nose, or eyes. It could also spread through contaminated surfaces. Current research shows that the transmission of the COVID-19 virus between people affects through direct, indirect, or generated contacts with people who will interfere with contaminated secretions such as saliva and respiratory secretions, people by rights, eliminate. , sneeze, talk or sing.

The United States is geographically an air distance of 7,236.93 miles from China. It is believed that the origin of the COVID-19 virus was in China. The way the COVID-19 virus spread from China to the US and hit the US, infected and killed people is by air. Human and electromagnetic waves need to be filled by their negative 
power. On the other hand, preventive measures should be followed by everyone and curable treatments should be administered to the infected. Around the United States, that is, on all of the United States' borders, including land and oceans, at all seaports and all airports, comprehensive security measures must be in place to prevent infection of our invisible enemy while we protect our nation from visible enemies until it is complete recovery.

Many vaccines are now available that should help make a speedy recovery. There is also research going on to invent powerful new vaccines with no side effects that will save people's lives. In this way, all the nations of the world can take preventive action to save the lives of the peoples of all nations by taking preventive action at the borders of the nations and all airports.

Future work will be to conduct more analysis using data set from the United States to find a solution to the current situation in the United States. To improve the quality of the adaptation to the data set, the parameters in the model function are increased. Similar big data analyzes are carried out in other countries to make decisions about preventive measures to save people's lives.

\section{Conclusion and suggestions}

On December 31, 2019, the WHO was informed about several infected cases of human respiratory diseases of unknown origin in Wuhan city of China, showing clinical symptoms similar to viral pneumonia, including a shortness of breath, dry cough, and severe fever. The WHO declared this epidemic as a global health emergency on January 20, 2020. [18]. The coronavirus epidemic began and is now spreading very rapidly in China around the world claiming more than 377,407 mass acute deaths and 6,365,625 infections confirmed as of June 2, 2020, at 2:20 am GMT is alerted by countries' ability to cope with an epidemic or pandemic.

Everyone in the world is afraid of acute deaths. From now on, it is the main duty and responsibility of researchers to give hope to living and active people. To expedite actions to save people's lives, mathematical models will help make decisions on major international public health issues and in reducing mortality using available resources during this time of the COVID-19 pandemic. The goal of this article aims to give a hope to better protect people from the disease-causing virus and to speed up the immediate general COVID-19 pandemic preparedness by predicting the lifetime of COVID-19 using three new mathematical models that depend on parameters. The parameters of the model work with the uncertainty of the model due to the current international real problem caused by the coronavirus.

The first model is a six-parameter model, and the second and third models are seven-parameter models. These three models are the generalizations of three models of Phem [75]. Errors due to models in this article are minimized by errors due to models from Phem. These three models can predict the number of out-of-date mass acute deaths and lifespan. To illustrate the applicability of the models, consider a large data set with a size of 54 days from February 29, 2020, to April 22, 2020, on the number of mass acute deaths in the United States. The main focus is on the United States due to significant infection and mass acute death from the coronavirus and, 
as a result, everyone's life is uncertain. Since this is a major public health medical problem with an accuracy of $95 \%$, results are obtained using three models. The mass acute death counts in the United States are fitted by the model functions of three models and a solution to an international health medical problem is found. Based on the acute death rate, the lifetime of the COVID-19 coronavirus is estimated to be 1484.76198616309920 days from February 29, 2020. That is, there will be no mass acute deaths from the COVID-19 virus in the United States after April 2024 if everyone follows the WHO guidelines and advice from health workers. The people and the government can prepare well for this situation and many lives can be saved. In [89], the life span of the United States is estimated to be 1464.7577891175067 days using a mathematical model.

A comparative study of all models is presented for various measures of error. The number of mass acute deaths in the United States outside the date of the 54day record is predicted using three models. The record is missing some counts during data collection and is identified. From the ratio of the standard deviation and the mass acute mean deaths, the total number of mass acute deaths for 54 days is predicted to be 62,969 . The calculated standard deviation around the line of regression shows that a large number is missing from the data set when collecting data from the United States. Using the coefficient of determination, the Model-C is predicted to provide $100 \%$ fitness with the given data set and only $0.0 \%$ change. All three models are eligible for the US dataset, but Model-C is the best of the three. Model-A, Model$\mathrm{B}$, and Model-C predict the total number of mass acute deaths over 54 days will be $66,557,67,085$, and 68,523, respectively. Since the best model is Model-C, the total number of mass acute deaths predicted for 54 days will be 68,523.

Currently, the best way to human is to fight against of the COVID-19 disease is to prevent the virus from spreading and to take individual protective measures and mass vaccinations. Many vaccines are now available in the world. With the help of vaccines, the disease can be prevented and the spread of the disease can be controlled in a short time like in Israel. Research is also being carried out to find new effective vaccines with no side effects and with great effectiveness in saving lives from different strains of the COVID-19 Virus. In this way, all the nations of the world can take preventive measures to save the lives of people of all nations by taking strict preventive measures at the borders of nations, including land and oceans, in all seaports and all airports must be carried out in full and preventive security measures taken to prevent infection of our invisible enemy while we protect our nation from visible enemies until full recovery.

At all borders of all nations, including land and oceans, in all seaports, and all airports, comprehensive and preventive security measures must be in place to prevent the infection of our invisible enemy like as we rotect our nation from visible enemies until full recovery.

If the epidemic is effectively stopped and ended, the virus will be eliminated. Social distancing, good personal hygiene, quick identification, and full quarantine, and mass vaccination are currently effective measures to reduce the rapid spread of the disease. Public health prevention measures for COVID-19, as well as the invention and manufacture of effective vaccines for different strains of COVID-19, help reduce new infections, complications, and deaths. 
Clinically reported from China, but we don't know if these people, who are not used to seeing doctors around the world, would be affected and died of the coronavirus and spread COVID-19 to others. Hence, all people should take good preventive measures. For these huge social, public, and economic ramifications of the new 2019 coronavirus epidemic prevention, everyone should wear a disposable face mask(N95). If you wear a face mask, you should know from health care workers, how to use it and when to remove and properly replace the mask promptly. Another important safety measure is wearing shoes to cover your feet and prevent infected sputum or fluid from leaking out of the nose and the affected person's mouth from falling to the floor. To avoid hand infection, cover your hands.

Above all, the top leaders, and the decision makers of all nations must carry out social, public, and economic reforms to save the people and raise the standards of the people again. Provide all effective vaccines, drugs, vitamins, minerals, nutritious foods to improve immunity, adequate COVID-19 testing facilities, clean water, protective clothing, face masks(N95), protective shoes, eye protection, all types of medical facilities, ambulances, and enough hospital beds, ventilators, life-saving medical oxygen in the nose, PPE-kits for health providers, AMBU bag and shelter for protection because our law says it is justice to save a life.

Future studies will be to conduct further analysis using data set from the United States to find a solution to the current situation in the United States. We plan to design models to improve the quality of fit to the dataset by fixing more parameters in the function (2) to take into account the uncertainty of the COVID-19 coronavirus in the population and further assumptions about the data on the infected population. Also, we plan to apply the models to other countries in the future.

Acknowledgements This part of the research was carried out at the University of Kerala, Kerala, India. All calculations in this document were performed on a Micro Vax II computer with mathematical precision by Matlab at Anna University, Chennai, India. I am very grateful to the unknown reviewers of this article and the editor of the journal.

\section{Conflict of interest}

The author of this article declares that he has no conflict of interest.

\section{Data availability statements}

The author of this article declares that the datasets of USA generated during and/or analysed during the current study are available in the [75,89] repository, https://doi.org/10.3390/math8050655, http://orcid.org/0000-0002-6122-3342, and https://www.worldometers.info/coronavirus/?utm ${ }_{c}$ ampaign = homeAdvegas 1 ?countries .

\section{Ethical approval}

The author of this article states that this article does not contain any research studies involving human or animal participants. 


\section{Funding details}

The author of this article states that this research was not funded by anyone.

\section{Authorship contributions}

Dr. K. Selvakumar developed an idea from his 34 years of research experience and wrote the original manuscript, including computer programming to produce graphic and digital results, and edited the full article in latex.

\section{References}

1. A.Adiga, D. Dubhashi, B. Lewis, M. Marathe, S. Venkatramanan, and A. Vullikanti, Mathematical Models for COVID 19 Pandemic: A Comparative Analysis, J. Indian In. Sci., 100(4), 793807 (2020).

2. P. Adhikari, S. Meng, Y.Wu, Y. Mao, R. Ye, Q.Wang, C. Sun, S. Sylvia, S. Rozelle, H. Raat, and H. Zhou, A scoping review of 2019 Novel Coronavirus during the early outbreak period: Epidemiology, causes, clinical manifestation and diagnosis, prevention and control, Res. Squ., 9(1),29. (March 17, 2020). https://doi.org/10.21203/rs.2.24474/v1

3. I. Ahmad, and S. M. Asad, Predictions of coronavirus COVID-19 distinct cases in Pakistan through an artificial neural network, Episode. Inf., 148(e222), 110 (2020). https://doi.org/10.1017/S0950268820002174

4. H. Akaike, Information theory and an extension of the maximum likelihood principle. In Proceedings of the Second International Symposium on Information Theory; Petrov, B.N., Caski, F., Eds. AkademiaiKiado: Budapest, Hungary., 267281 (1973).

5. D.H. Alene, and W. Yadeta, Outbreak steps in the case of 2019 novel coronavirus infection, a review. Ann. Ant., Anti., 4(1), 1-7 (2020). https://doi.org/10.17352/aaa.000007

6. H. Algahtani, A. Subahi, and B. Shirah, Neurological Complications of Middle East Respiratory Syndrome Coronavirus: A Report of Two Cases and Review of the Literature, Ca. Rep. Neuro. Med., 1-6 (2016). https://doi.org/10.1155/2016/3502683

7. H. Ali, F. Hossain, M. Hasan, and S. Abuja, Covid-19 Dataset: Worldwide spread log including countries first case and the first death, Data in Brief 32 (106173), 1-7 (2020) https://doi.org/10.1016/j.dib.2020.106173

8. A.R. Appadu, A.S. Kelli, and Y.O. Tijani, Comparison of some forecasting methods for COVID-19, Alexandria Eng., 60, 15651589 (2021). https://doi.org/10.1016/j.aej.2020.11.011

9. A. Asraf, Z. Islam, R. Haque, and M. Islam, Deep Learning Applications to Combat Novel Coronavirus (COVID 19) Pandemic, SN. Comp. Sci., 1(363), 1-7 (2020). https://doi.org/10.1007/s42979020-00383-w

10. R. Azamfirei, The 2019 Novel Coronavirus: A Crown Jewel of Pandemics?, Cri. Ca. Med., 6(1), 3-4 (2020). https://doi.org/ 10.2478/ccm-2020-0013

11. A. Banerjee, L. Paseo, S. Harris, A. Gonzalez-Izquierdo, A. Torralbo, L. Shallcross, M. Noursadeghi, D. Pillay, N. Sebire, C. Holmes, C. Pagel, W. K. Wong, C. Langenberg, B. Williams, S. Texas, and H, Hemingway, Estimating excess 1-year mortality associated with the COVID-19 pandemic according to underlying conditions and age: a population-based cohort study, The lancet 395, 1715-1725 (2020). https://www.thelancet.com

12. A. L. Bertozzi, E. Franco, G. Mohler, M. B. Short, and D. Sledge, The challenges of modeling and forecasting the spread of COVID-19, PNAS, 117(29), 1673216738 (July 21, 2020).

13. S. K., Biswas, J. K. Ghosh, S. Sarkar, and U. Ghosh, COVID-19 pandemic in India: a mathematical model study, Nonlinear Dyn, 102, 537553 (2020). https://doi.org/10.1007/s11071-020-05958-Z

14. T. Z. Boulmezaoud, A discrete epidemic model and a zigzag strategy for curbing the covid-19 outbreak and for lifting the lockdown Math. Model. Nat. Phenom. 15 (75), 1-34 (2020).

15. M. N. K. Boulos 1 and E. M. Geraghty, Geographical tracking and mapping of coronavirus disease COVID19/severe acute respiratory syndrome coronavirus (SARSCoV2) epidemic and associated events around the world: how 21st century GIS technologies are supporting the global fight against outbreaks and epidemics, Int. J. Heal. Geogr., 19(8), 1-13 (2020). https://doi.org/ 10.1186/s12942020-00202-8 
16. J. Breda, and C. F. Borges, Preparedness, prevention and control of COVID-19 in prisons and other places of detention Interim guidance, WHO, Regional Office for Europe, Case 2:90-cv-00520KJMDB Document 6529-2 Filed 03/25/20, 1-6 (15 March 2020).

17. M. Choisy, J, F. Gugan, and P. Rohani, Mathematical Modeling of Infectious Diseases Dynamics, Encyclopedia of Infectious Diseases: Modern Methodologies, by M.Tibayrenc, Chapter 22, 379-404 (2020).

18. M. Chung, A. Bernheim, X. Mei, N. Zhang, M. Huang, X. Zeng, J. Cui, W. Xu, Y. Yang, Z.A. Fayad, A. Jacobi, K. Li, S. Li, and H. Shan, CT Imaging Features of 2019 Novel Coronavirus (2019-nCoV), Radi., 295(/1), 202-207 (April 2020). https://doi.org/ 10.1148/Radiol.2020200230

19. Z. Chen, One thought on COVID-19 and Emerging Viral Diseases: the journey from animals to humans, Hraward University ( March 22, 2020). http://sitn.hms.harvard.edu

20. T. M. Chen, J. Rui, Q. P. Wang, Z. Y. Zhao, J. A. Cui, and L. Yin, A mathematical model for simulating the phase-based transmissibility of a novel coronavirus Infec. Dis. Pov., 9(24), 1-8 (2020). https://doi.org/10.1186/s40249-020-00640-3

21. R. Cherniha, and V. Davydovych, A Mathematical Model for the COVID-19 Outbreak and Its Applications, Sym., 12(990), 1-12 (2020). https://doi.org/10.3390/sym12060990, www.mdpi.com/journal/symmetry

22. T. M. Chen, J. Rui, Q. P. Wang, Z. Y. Zhao, J. A. Cui, and L. Yin, A mathematical model for simulating the phase-based transmissibility of a novel coronavirus Infec. Dis. Pov., 9(24), 1-8 (2020). https://doi.org/10.1186/s40249-020-00640-3

23. J. D. Cherry, and P. Krogstad, SARS: The First Pandemic of the 21st Century, Pediatric Research, 56( 1), 1-5 (2001). http://dx.doi.org/ 10.1203/01.PDR.0000129184.87042.FC

24. J. Cleland, E. C. P. Tan1, K. Y. Tham1, and N. L. Beer1 How Covid-19 opened up questions of sociomateriality in healthcare education, Ad. Heal. Sci. Edu., 25, 479482 (2020). https://doi.org/ 10.1007/s10459-020-09968

25. S. Dhamodharavadhan, R. Rathipriya, and J.M. Chatterjee, COVID-19 Mortality Rate Prediction for India Using Statistical Neural Network Models. Front. Public Health, 8(441), 1-12 (2020). https://doi.org/10.3389/fpubh.2020.00441

26. E. El-Shafeiy, A. E. Hassanien, K.M. Sallam, and A. A. Abohany, Approach for Training Quantum Neural Network to Predict Severity of COVID-19 in Patients, CMC,66(2), 1745-1755 (2021) https://doi.org/10.32604/cmc.2020.013066

27. A.S. Fokas, N. Dikaios, and G. A. Kastis, Mathematical models and deep learning for predicting the number of individuals reported to be infected with SARS-CoV-2, J. R. Soc. Interface 1720200494), 1-10 (2020). http://dx.doi.org/10.1098/rsif.2020.0494

28. S. Ghisolfi, I. Alms, J.C. Sandefur, T.V Carnap, J. Heitner, and T. Bold, Predicted COVID-19 fatality rates based on age, sex, comorbidities, and health system capacity, BMJ Global Health, 5:e003094. 1-8 (2020). https://doi.org/ 10.1136/bmjgh-2020-003094

29. H. S. Gopalan, and A. Misra, COVID-19 pandemic and challenges for socio-economic issues, healthcare, and National Health Programs in India, Diabetes Metabolic Syndrome: Clinical Research Reviews 14, 757-759 (2020).

30. S. Gupta, G. S. Raghuwanshi, and A. Chanda, Effect of weather on COVID-19 spread in the US A prediction model for India in 2020, Science of the Total Environment 728 (138860), 1-8 (2020). https://doi.org/10.1016/j.scitotenv.2020.138860

31. K. D. Gupta, R. Dwivedi, and D. K. Sharma, Prediction of Covid-19 trends in Europe using generalized regression neural network optimized by flower pollination algorithm, J. Interim. Math., 1-19 (2020). http://dx.doi.org/ 10.1080/09720502.2020.1833447

32. A.S. Fokas, N. Dikaios, and G.S. Kastis, Mathematical models and deep learning for predicting the number of individuals reported to be infected with SARS-CoV-2. J. R. Soc. Interface 17(20200494) 1-13 (2020). http://dx.doi.org/10.1098/rsif.2020.0494

33. N. M. Ghazaly, M. A. Abdel-Fattah, and A. A. A. El-Aziz, Novel Coronavirus Forecasting Model using Nonlinear Autoregressive Artificial Neural Network, In. J. Advan. Sci. Tech., 29(5s), 18311849 (2020). https://www.researchgate.net/publication/340538849

34. Y. Hao, T. Xu, H. Hu, P. Wang, and Y. Bai, Prediction and analysis of Corona Virus Disease 2019. PLoS ONE 15(10): e0239960. 1-15 (2020). https://doi.org/10.1371/journal.pone.0239960

35. H. W. Hethcote, The Mathematics of Infectious Diseases, SIAM Review, 42(4), 599-653 (Dec. 2000).

36. E. Irmak, Implementation of convolutional neural network approach for COVID-19 disease detection, Physiol Genomics, 52, 590601 (2020). http://dx.doi.org/10.1152/physiolgenomics.00084.2020 
37. T. P. Jackson, J. J C King, C. Makungu, N. Spieker, S. Woodd, P. Risha, and C. Goodman, Infection prevention and control compliance in Tanzanian outpatient facilities: a cross-sectional study with implications for the control of COVID-19, Lan. Glo. Heal., 1-10 ( May 6, 2020 ). https://doi.org/ 10.1016/S2214-109X(20)30222-9

38. M. Jamshidi, A.Albakhsh, J. Talla, Z..K Peroutka, F. Hadjilooei, P. lalbakhsh, M. Jamshidi, L.L. Spada, M. Mirmozafari, M. Dehghani, A. Sabet, S Roshani, S. Roshani, N. Bayat-Makou, B. Mohamadzade, Z Malek, AJamshidi, Sarah Kiani1, L. Hashemi-Dezaki, and W. Mohyuddin, Artificial Intelligence and COVID-19: Deep Learning Approaches for Diagnosis and Treatment, 8(109595), 1-14, (2020). http://dx.doi.org/10.1109/ ACCESS.2020.3001973

39. N. P. Jewell, J. A. Leonard, and B. L. Jewell, Predictive Mathematical Models of the COVID-19 Pandemic Underlying Principles and Value of Projections, JAMA, 323(19), 1893 (May 19, 2020). https:/ jamanetwork.com/ on 11/24/202

40. R.G.Joshua, and D.L. Ronald, Demographic perspectives on the mortality of COVID-19 and other epidemics, Proc. Natl. Acad. Sci. U.S.A. 117, 2203522041 (August 20, 2020). https://doi.org/10.1073/pnas.2006392117

41. R.G.Joshua, and D.L. Ronald, Demographic perspectives on the mortality of COVID19 and other epidemics, PNAS, 117(36), 2203522041 (September 8, 2020). https:/ www.pnas.org/cgi/doi/10.1073/pnas.2006392117

42. D. Kasilingam, S.P. Sathiya Prabhakaran, D.K. Rajendran, V. Rajagopal, T. Santhosh Kumar, and A. Soundararaj. Exploring the growth of COVID-19 cases using exponential modeling across 42 countries and predicting signs of early containment using machine learning. Transbound Emerg Dis., 1-18 (2020). http://dx.doi.org/10.1111/tbed.13764

43. B. Khajji, D. Kada, O. Balatif, and M. Rachik A multi-region discrete-time mathematical modeling of the dynamics of Covid-19 virus propagation using optimal control, Appl. Math. Comput. 64:255281 (2020). https://doi.org/10.1007/s12190-020-01354-3

44. S. Kikkisetti, J. Zhu, B. Shen, H. Li, and T.Q. Duong, Deep-learning convolutional neural networks with transfer learning accurately classify COVID-19 lung infection on portable chest radiographs PeerJ 8:e10309, 1-13 (2020). https://doi.org/10.7717/peerj.10309

45. J. D. Cherry and P. Krogstad, SARS: The First Pandemic of the 21st Century, Ped. Res., 56, 1-6 (2004). https://doi.org/ 10.1203/01.PDR.0000129184.87042.FC

46. D.M. Levine, D. Stephan, T.C.Krehbiel, and M.L.Berenson, Statistics for Managers Using Microsoft Excel, Pearson Prentice Hall, (2009).

47. Q. Li, and H. Pham, NHPP Software Reliability Model Considering the Uncertainty of Operating Environments With Imperfect Debugging and Testing Coverage. Appl. Math. Model., 51, 6885 (2017).

48. A. S. Lozano, F. C. Boyero, A. L. Jimnez, C. C. Felgueroso, A. C. Garca, P. L. Valenzuela, J. Arenas, A. Lucia*, M. A. Martn and COVID-19 Hospital 12 Octubre Clinical Biochemisty Study Group, Can routine laboratory variables predict survival in COVID-19? An artificial neural network-based approach, Clin Chem Lab Med; 58(12), e299e302 (2020). (https://doi.org/10.1515/cclm-2020-0730

49. A.Makris, I. Kontopoulos, and, K. Tserpes, COVID-19 detection from chest X-Ray images using Deep Learning and Convolutional Neural Networks, SETN 2020: 11th Hellenic Conference on Artificial Intelligence, 6066 (2020). https://doi.org/10.1145/3411408.3411416

50. H. Miao, Q. Gao, H. Feng, C. Zhong, P. Zhu, L. Wu, M.D. Swartz, X. Luo, S.M. DeSantis, D. Lai D, C. Bauer, A. Prez, L. Rong, and D. Lairson, Mathematical Modeling of Business Reopening When Facing SARS-CoV-2 Pandemic: Protection, Cost, and Risk. Front. Appl. Math. Stat. 6(35), 1-13 (2020). https://doi.org/10.3389/fams.2020.00035

51. P. Mishra, C. Fatih, D. Rawat, S. Sahu, S.A. Pandey, M. Ray, A. Dubey, and O. M. Sanusi, Trajectory of COVID-19 Data in India: Investigation and Project Using Artificial Neural Network, Fuzzy Time Series and ARIMA Models, Annual Research Review in Biology, 35(9), 46-54 ( 2020).

52. Y. Mohamadou, A. Halidou, and P.T. Kapen, A review of mathematical modeling, artificial intelligence, and datasets used in the study, prediction, and management of COVID-19, Appl. Intel., 50, 39133925 (2020). https://doi.org/10.1007/s10489-020-01770-9

53. A.Mollalo, K. M. Rivera, and B. Vahedi, Artificial Neural Network Modeling of Novel Coronavirus (COVID-19) Incidence Rates across the Continental United States, Int. J. Environ. Res. Public Health, 17(4204), 1-13 (2020). https://doi.org/10.3390/ijerph17124204, www.mdpi.com/journal/ijerph

54. M.C. Muttrack, and R. Scherhov, Assessing the potential impact of COVID-19 on life expectancy, PLoS ONE, 15(9), e0238678 (2020). https://doi.org/10.4371/journal.pone.0238678

55. Nash .C. Mediaite, Hardware Professor Sounds Alarmed on Likely Coronavirus Pandemic. $40 \%$ to $70 \%$ of the World could be Infected this year. https://www.mediaite.com/news/harward-professor- 
sounds alarm on likely coronavirus pandemic -. 40 to - 70 of world could be - infected this year

56. I. Nesteruk, statistics-based predictions of coronavirus epidemic spreading in mainland china, Innov Biosyst Bioeng, 4(1), 1318 (2020). https://doi.org/ 10.20535/ibb.2020.4.1.195074 UDC 519.2, 519.8

57. I. Nesteruk, Simulations and predictions of the covid-19 pandemic with the use of sir model, Innov Biosyst Bioeng, 4(2), 110121 (2020). https://doi.org/ 10.20535/IBB.2020.4.2.204274 UDC 519.2, 519.8

58. H. R. Niazkar, and M. Niazkar, Application of artificial neural networks to predict the COVID-19 outbreak, Global Health Research and Policy, 5(50), 1-11 (2020). https://doi.org/10.1186/s41256020-00175-y

59. R. Pal, A. A. Sekh, S. Kar, and D. K. Prasad, Neural Network-Based Country Wise Risk Prediction of COVID-19, Appl. Sci. 5(2004.00959v2), 1-18 (2020). https://doi.org/10.3390/appxx010005, www.mdpi.com/journal/applsci

60. Y. Park, D. Casey, I. Joshi, J. Zhu, and F. Cheng, Emergence of New Disease: How Can Artificial Intelligence Help?, Trends in Molecular Medicine, 26(7), 627 (2020).

61. S M Peiris, Y Guan KY Yuen, Severe acute respiratory syndrome, Nat. Med. Suppl., 10(12), (Dec2004).

62. M. Perc, N. G. Miksic, M. Slavinec, and A. Stoer, Forecasting COVID-19, Front. Phys. 8(127), 1-5 ( 2020). https://doi.org/10.3389/fphy.2020.00127

63. G,. Pereira , J. M. Guerin , A, G. S. Jnior, G. S. Garcia , P. Piscitelli, A. Miani , C. Distante, and L.M. G. Gonalves, Forecasting Covid-19 Dynamics in Brazil: A Data Driven Approach, Int. J. Environ. Res. Public Health, 17 (5115) , 1-26 (2020). https://doi.org/10.3390/ijerph17145115 , www.mdpi.com/journal/ijerph

64. G. Perone, ARIMA forecasting of COVID-19 incidence in Italy, Russia, and the USA, 1-20 (2020). https://ssrn.com/abstract $=3612402$

65. F. Petropoulos, and S. Makridakis, Forecasting the novel coronavirus COVID-19, PLoS ONE, 16(3), e0231236 (2020). https://doi.org/10.1371/journal.pone.0231136

66. H. Pham, D. H. Pham, and H. Pham Jr. A New Mathematical Logistic Model, and Its Applications. Int. J. Inf. Manag. Sci., 25, 7999 (2014).

67. H. Pham, Modeling, U.S. Mortality and Risk-Cost Optimization on Life Expectancy. IEEE Trans. Reliab., 60, 125133 (2011).

68. H. Pham, A New Criteria for Model Selection. Mathematics., 7, 1215 (2019).

69. H. Pham, A Logistic Fault-Dependent Detection Software Reliability Model. J. Univers. Comput. Sci., 24, 17171730 (2018).

70. H. Pham, System Software Reliability., Springer: London, UK, 2006)

71. T. Pham, and H.A. Pham, Generalized Software Reliability Model with Stochastic Fault-detection Rate. Ann. Oper. Res.. 277, 8393 (2019).

72. H. Pham, and D.H. Pham, A Novel Generalized Logistic Dependent Model to Predict the Presence of Breast Cancer Based on Biomarkers. Concurr. Comput. Pract. Exp.. 32, e5467 (2020),

73. T. D. Pham, A comprehensive study on classification of COVID-19 on computed tomography with pre-trained convolutional neural networks, Scientific Reports, 10(16942), 1-7 (2020). https://doi.org/10.1038/s41598-020-74164-z

74. Q.V. Pham, D.C. Nguyen, T. H. The, W.J. Hwang, and P.N. Pathirana, Artificial Intelligence (AI) and Big Data for Coronavirus (COVID-19) Pandemic: A Survey on the State-of-the-Arts, 1-17 (2020). https://doi.org/10.20944/preprints202004.0383.v1

75. H. Pham, On Estimating the Number of Deaths Related to Covid-19, Math., 8, 655, 1=9 (2020). https://doi.org/10.3390/math8050655, http://www.mdpi.com/journal/mathematics

76. C. Pongkitivanichkul, D. Samart1, T. Tangphati, P. Koomhin, P. Pimton,6, P. Dam-O, A. Payaka, and P. Channuie, Estimating the size of COVID-19 epidemic outbreak, Phys. Scr. 95 (085206), 1-7 (2020). https://doi.org/10.1088/1402-4896/ab9bdf

77. B. Prasse, M. A. Achterberg, L. Ma, and P. V. Mieghem, Network-inference-based prediction of the COVID-19 epidemic outbreak in the Chinese province Hubei, Appl. Network Sci., 5(35), 1-11 (2020). https://doi.org/10.1007/s41109-020-00274-2

78. Z. Qiao, A. Bae, L.M. Glass, C. Xiao, and J. Sun, FLANNEL (Focal Loss bAsed Neural Network EnsembLe) for COVID-19 detection, American Medical Informatics Association, 00(0), 1-9 (2020). https://doi.org/10.1093/jamia/ocaa280

79. F. A. Rabi, M. S. Al Zoubi, G. A. Kasasbeh, D. M. Salameh, and A. D. Al-Nasser, SARS-CoV-2 and Coronavirus Disease 2019: WhatWe Know So Far, Patho., 9, 231 (2020). http://www.mdpi.com/journal/pathogens 
80. A. Rdulescu, C. Williams, and K. Cavanagh, Management strategies in an SEIR type model of COVID 19 community spread Sci. Rep., 10(21256), 1-14 (2020). https://doi.org/10.1038/s41598-020-77628-4

81. M. Rahimzadeh, and A. Attar, A modified deep convolutional neural network for detecting COVID-19 and pneumonia from chest X-ray images based on the concatenation of Xception and ResNet50V2, Inform, in Medi. Unlock., 19 (100360), 1-9 (2020). https://doi.org/10.1016/j.imu.2020.100360, http://www.elsevier.com/locate/imu

82. J. Rasheed, A. A. Hameed, C. Djeddi, A. Jami, and F. Al Tudjman, A machine learning-based framework for the diagnosis of COVID 19 from chest X-ray images, Interim. Sci. Comput. Life Sci., 1-17 (2020). https://doi.org/10.1007/s12539-020-00403-6

83. M. E. Rosti, S. Olivieri, M. Cavaiola, A. Seminara, and A. Mazzino, Fluid dynamics of COVID-19 airborne infection suggests urgent data for a scientific design of social distancing, Sci. Rep., 10(22426), 1-9 (2020) https://doi.org/10.1038/s41598-020-80078-7

84. R. Sameni, Mathematical Modeling of Epidemic Diseases; A Case Study of the COVID-19 Coronavirus, https://arxiv.org/abs/2003.11371

85. T. Santra, Fitting mathematical models of biochemical pathways to steady-state perturbation response data without simulating perturbation experiments, Sci. Rep. - 8(11679), 1-14 (2020) https://doi.org/10.1038/s41598-018-30118-0

86. G. Schwarz, Estimating the dimension of a model. Ann. Stat., 6, 461464 (1978).

87. E.D. L. Scherf, M. V. V. Silva, and J. S. Facchini, The Management (or Lack Thereof) of COVID-19 in Brazil: Implications for Human Rights Public Health, Human Rights in Healthcare. 1-24 (2020). https://www.emerald.com/insight/publication/issn/2056-4902.

88. B. Sekeroglu, and I. Ozsahin, Detection of COVID-19 from Chest X-Ray Images Using Convolutional Neural Networks, SLAS Technology, 25(6) 553565 (2020). https://doi.org/10.1177/2472630320958376

89. K. Selvakumar, and S. Lokesh, The prediction of the lifetime of the new coronavirus in the USA using mathematical models, Soft Computing 1-20 (2021). http://orcid.org/0000-0002-6122-3342

90. M. Serhani, and H. Labbardi, Mathematical modeling of COVID-19 spreading with asymptomatic infected and interacting peoples, App. Math. Comput., 1-25 (August 2020). https://doi.org/10.1007/s12190-020-01421-9

91. G. R. Shinde, A.B. Kalamkar, P.N. Mahalle, N. Dey, J. Chaki, and A., E. Hassanien, Forecasting Models for Coronavirus Disease (COVID 19): A Survey of the State of the Art, SN Computer Science, 1(197), 1-15 (2020). https://doi.org/10.1007/s42979-020-00209-9

92. R. K. Singh, M. Rani, A.S. Bhagavathula, Ranjit Sah, A.J.R. Morales, H. Kalita, C. Nanda, S Sharma, Y.D. Sharma, A.A. Rabaan, J. Rahmani, and Pavan Kumar, Prediction of the COVID19 Pandemic for the Top 15 Affected Countries: Advanced Autoregressive Integrated Moving Average (ARIMA) Model, JMIR Public Health Surveill, 6(2), e19115 (2020). https:// publichealth.jmir.org/2020/2/e19115

93. D. Singh, V. Kumar, V. Yadav, and M. Kaur, Deep Convolutional Neural Networks based Classification model for COVID-19 Infected Patients using Chest X-ray Images, Int. J. Pattern Recognition and Artificial Intelligence, 1-18 (2020). https://doi.org/10.1142/S0218001421510046, https://doi.org/10.1142/S0218001421510046

94. F. Song, N. Shi, Fei Shan, Z. Zhang, J. Shen, H. Lu, Y. Ling, Y. Jiang, and Y. Shi, Emerging 2019 Novel Coronavirus (2019-nCoV), Pneu. Rad., 295(1), 210-217, (Apl 2020). https://doi.org/ 10.1148/Radiol.2020200274

95. M. Sharma, V.B. Singh, and H. Pham, H, Entropy-Based Software Reliability Analysis of MultiVersion Open Source Software. IEEE Trans. Softw. Eng., 44, 12071223 (2018).

96. K. Stadler, V. Masignani, M. Eickmann, S. Becker, S. Abrignani, H-D. Klenk and R. Rappuoli, SARS Beginning to understand a new virus, Nat. Rev., Micro., 1, 209-218 (Dec 2003).

97. S.K. Tamang, P.D. Singh, and B. Datta, Forecasting of Covid-19 cases based on prediction using artificial neural network curve fitting technique, Global J. Environ. Sci. Manage. 6(SI): 53-64 (2020). https://doi.org/10.22034/GJESM.2019.06.SI.06, https://www.gjesm.net/article39823.html

98. A. Tavakoli, K. Vahdat and M. Keshavarz, Novel Coronavirus Disease 2019 (COVID-19): An Emerging Infectious Disease in the 21st Century, Iran South Med. J, 22(6): 432-450 (2020).

99. M. I. Uddin, S. A. A. Shah, and M, A. Al-Khasawneh, A Novel Deep Convolutional Neural Network Model to Monitor People following Guidelines to Avoid COVID-19, J. Sensors, 8856801, 1-16 (2020). https://doi.org/10.1155/2020/8856801

100. D. F. Verachi, L. Trussoni, and L. Lanzi, COVID-19 in Italy: a mathematical model to analyze the epidemic containment strategy and the economic impacts, Risk Management Magazine ANNO 15(2) - PAGINA - 23 -34 (2020). 
101. M.T. Vrugt, J. Beckmann, and R. Wittkowski, Effects of social distancing and isolation on epidemic spreading modeled via dynamical density functional theory, Nature Communications, 11(5576), 1-11 (2020). https://doi.org/10.1038/s41467-020-19024-0, —www.nature.com/naturecommunications

102. P. Verhulst, Recherches mathematiques sur la loi d'accroissement de la population. Nouv. Mem. de l'Academie Royale des Sci. et Belles-Lettres de Bruxelles., 141 (1845).

103. P. Vkovski, A. Kratzer, S. Steiner, H. Stalder, and Volker Thiel, Coronavirus biology and replication: implications for SARS- CoV-2, Nature Reviews, Microbiology, 1-16 (2020). https://coronavirus.jhu.edu/map.html

104. M. Wang, Y. Zhou, Z. Zong, Z. Liang, Yu Cao, H. Tang, B. Song, Z. Huang, Y. Kang, P. Feng, B. Ying, and W. Li3, A precision medicine approach to managing 2019 novel coronavirus pneumonia, Pre. Clin. Med., 3(1), 1421 (2020). https://doi.org/ 10.1093/PC media/pbaa002

105. L. Wang, Z. Q. Lin, and A. Wong, COVID-Net: a tailored deep convolutional neural network design for detection of COVID-19 cases from chest X-ray images, Scientific Reports, 10(19549), 1-11 (2020). https://doi.org/10.1038/s41598-020-76550-z

106. L. Wynants, B. V. Clster, G. S., Collins, R. Driley, G. Heinze, E. Schuit, M. M. J. Bonten, D. L. Dahly, J. A.A. Damen, T. P. A. Debray, V. M. T. De Jong, M. D. Vos, P. Dhiman, M. C. Haller, M. O. Harhay, L. Henckaerts, P. Hues, N. Kreuzberger, A. Lohmann, K. Luijken, J. Ma, G. P.Martin, C. L. A. Navarro, J. B. Reitsma, J. C. Sergeant, C. Shi, N. Skoetz, I. J. M. Smits, K. I.E. snell, M. Sperrin, R. Spijker, E.W. Steyerberg, T. Takada, I. Tzoulaki, S. M. J. V. Kuijk, F. S. V. Royen, J. Y. Verbakel, C. Wallisch, J. Wilkinson, R. Wolff, L. Hooft, K. G M. Moons, and M. V. Smeden, Prediction models for diagnosis and prognosis of covid-19: systematic review and critical appraisal, BMJ, 369(1328), 1-24 ( 2020). https://doi.org/10.1136/bmj.m1328

107. Y.N. Kyrychko, K. B. Blyuss, and I. Brovchenko, Mathematical modeling of the dynamics and containment of COVID 19 in Ukraine Sci. Rep., 10(19662), 1-11 (2020). https://doi.org/10.1038/s41598020-76710-1

108. O. Zakary, S. Bidah, M. Rachik, and H. Ferjouchia, Mathematical Model to Estimate and Predict the COVID-19 Infections in Morocco: Optimal Control Strategy, J. Appl. Math. , Hindawi, 1-13 (2020). https://doi.org/10.1155/2020/9813926

109. A.Zeb, E. Alzahrani, V. S. Erturk, and G. Zaman, Mathematical Model for Coronavirus Disease 2019 (COVID-19) Containing Isolation Class, BioMed Research International, 3452402, 1-7 (2020). https://doi.org/10.1155/2020/3452402

110. Y. Zhang, C. You, Z. Cai, J. Sun, W. Hu, and X. H. Zhou, Prediction of the COVID 19 outbreak in China based on a new stochastic dynamic model, Sci, Rep., 10(21522), 1-9 (2020). https://doi.org/10.1038/s41598-020-76630-0

111. Z. Zhang, A. Zeb, S. Hussain, and E. Alzahrani, Dynamics of COVID-19 mathematical model with stochastic perturbation Advances in Difference Equations, 451, 1-12 (2020). https://doi.org/10.1186/s13662-020-02909-1

112. M. Zhu, and H. Pham, A Software Reliability Model Incorporating Martingale Process with GammaDistributed Environmental Factors. Ann. Oper. Res., (2018).

113. Y. Zhou, F. Wang, J. Tang, R. Nussinov, and F. Cheng, Artificial intelligence in COVID-19 drug repurposing, Digital-healtH, 2, 2667-2676 (2020). www.thelancet.com/digital-health Vol 2 December 2020

114. H. Zhu, Y. Li, X. Jin, J. Huang, X. Liu, Y. Qian, and J. Tan, Transmission Dynamics and Control Methodology of COVID-19: a Modeling Study, (April 1, 2020). https://doi.org/10.1101/2020.03.29.20047118

115. M. Zuo, S. K. Khosa, Z. Ahmad, and Z. Almaspoor, Comparison of COVID-19 Pandemic Dynamics in Asian Countries with Statistical Modeling, Comput., Math. Meth. Med., 4296806, 1-16 (2020). https://doi.org/10.1155/2020/4296806

116. Centers for Disease Control and Prevention. ( 8 April 2020). https://www.cdc.gov/coronavirus/2019ncov/prevent-getting-sick/social-distancing.html

117. Coronavirus Death Toll and Trends Worldometer, (2020). www.worldometers.info coronavirus

118. Experts explain the latest bulletin of unknown causes of viral pneumonia, WCHC (14 Jan 2020). http://wjw.wuhan.gov.cn/front/web/showDetail/2020011109036

119. GenBank. Wuhan seafood market pneumonia virus isolates Wuhan-Hu-1, complete genome (16 Jan 2020). https://www.ncbi.nlm.nih.gov/nuccore/MN908947

120. Health Commission of Guangdong Province. Our province actively responds to the pneumonia epidemic of new coronavirus infection (20 Jan 2020). http://wsjkw.gd.gov.cn/zwyw..yqxx/content/post..2876926.html 
121. Holmes E. Initial genome release of novel coronavirus (14 Jan 2020). http://virological.org/t/initialgenome- release-of-novel-coronavirus/319

122. MERS-CoV case in England, Public Health England (PHE) confirm that an individual has been diagnosed with Middle East Respiratory Syndrome (MERS) in England, Press release, (2019).

123. Modeling COVID-19 scenarios for the United States, IHEM COVID-19 Forecasting Team Nature Medicine, (2020). https://doi.org/10.1038/s41591-020-1132-9

124. News X. Experts claim that a new coronavirus is identified in Wuhan (14 Jan 2020). http://www.xinhuanet.com/2020-01/09/c..1125438971.htm

125. Novel Coronavirus (2019-nCoV) Twitter Message, WHO Regional Office for Western Pacific (20 Jan 2020). https://twitter.com/WHOWPRO/status/1219478541865144320

126. Rapid Risk Assessment: Outbreak of acute respiratory syndrome associated with a novel coronavirus, Wuhan, China; first update 22 January 2020. ECDC: Stockholm (2020).

127. Rapid Risk Assessment: Cluster of pneumonia cases caused by a novel coronavirus, ECDC, Wuhan, China (17 Jan 2020). https://www.ecdc.europa.eu/en/publications-data/rapid-riskassessment-clusterpneumonia- cases-caused-novel-coronavirus-Wuhan

128. SARS and MERS, Baylor College of Medicine, Houston, Texas. https://www.bcm.edu/departments/molecular-virology-and-microbiology/emerging-infectionsandbiodefense/sars-virus

129. The epidemic situation of new coronavirus infection on January 21, 2020, National Health Commission of the Peoples Republic of China. (21 Jan 2020) http://www.nhc.gov.cn/yjb/s3578/202001/930c021cdd1f46dc832fc27e0cc465c8.shtml

130. Wuhan Municipal Health and Health Commissions briefing on the current pneumonia epidemic situation in our city, WCHC ( 14 Jan 2020). http://wjw.wuhan.gov.cn/front/web/showDetail/2019123108989

131. Wuhan Municipal Commission of Health and Health on pneumonia of new coronavirus infection, WCHC (16 Jan 2020). http://wjw.wuhan.gov.cn/front/web/showDetail/2020011609057

132. Wuhan Municipal Commission of Health on pneumonia of new coronavirus, WCHC (16 Jan 2020). http://wjw.wuhan.gov.cn/front/web/showDetail/2020011509046

133. Wuhan Municipal Commission of Health on pneumonia of new coronavirus infection, WCHC (20 Jan 2020). http://wjw.wuhan.gov.cn/front/web/showDetail/2020012009077

134. Wuhan Municipal Commission on Health on pneumonia of new coronavirus infection, WCHC (21 Jan 2020). http://wjw.wuhan.gov.cn/front/web/showDetail/2020012109083

135. Xinhua Net. Xi orders resolute efforts to curb virus spread ( 20 Jan 2020). http://xinhuanet.com/english/2020-01/20/ c138721535:htm 
Figures

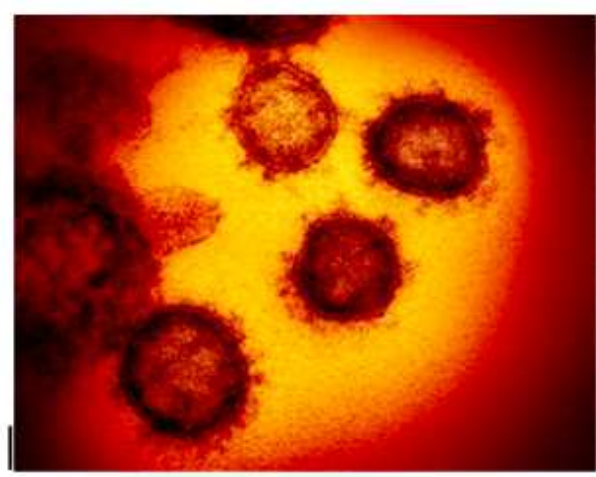

a

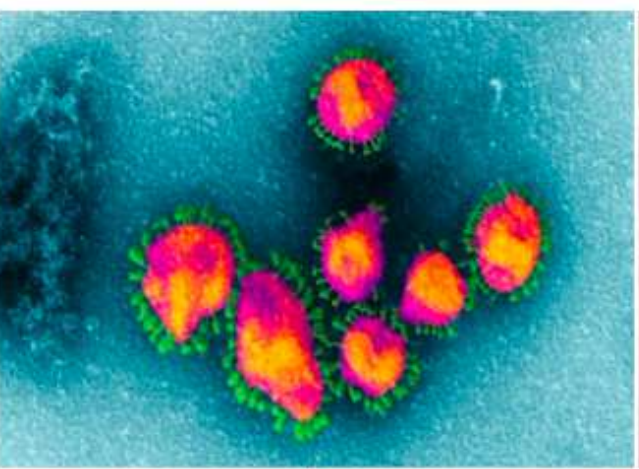

b

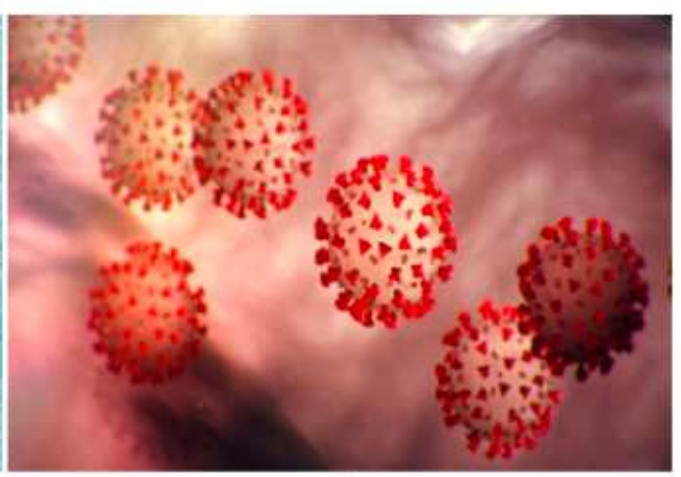

C

\section{Figure 1}

a). SARS-CoV, b). MERS-CoV, and c). SARS-CoV-2 with crowns

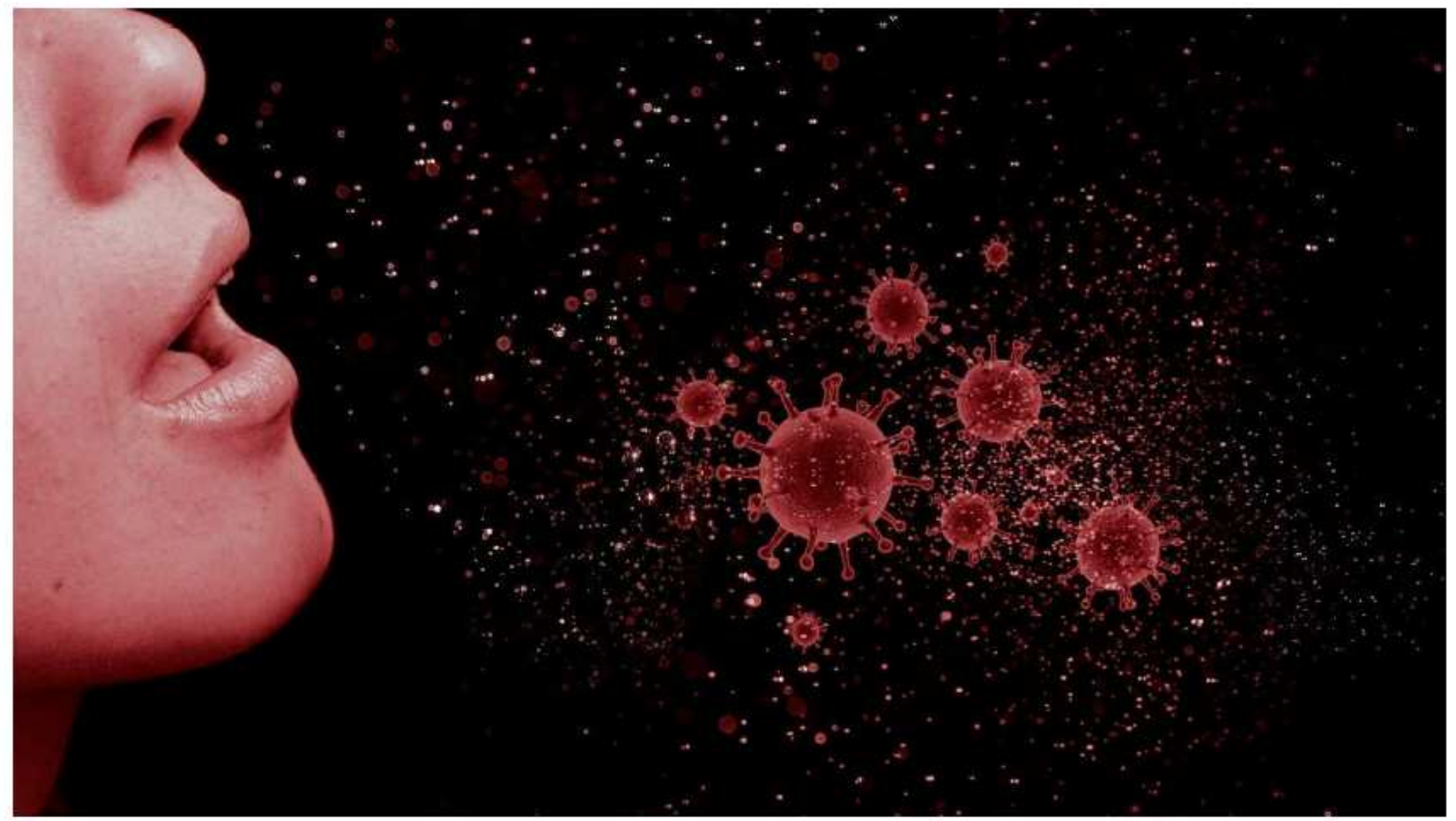

\section{Figure 2}

The spread of COVID-19 virus from an infected patient. 


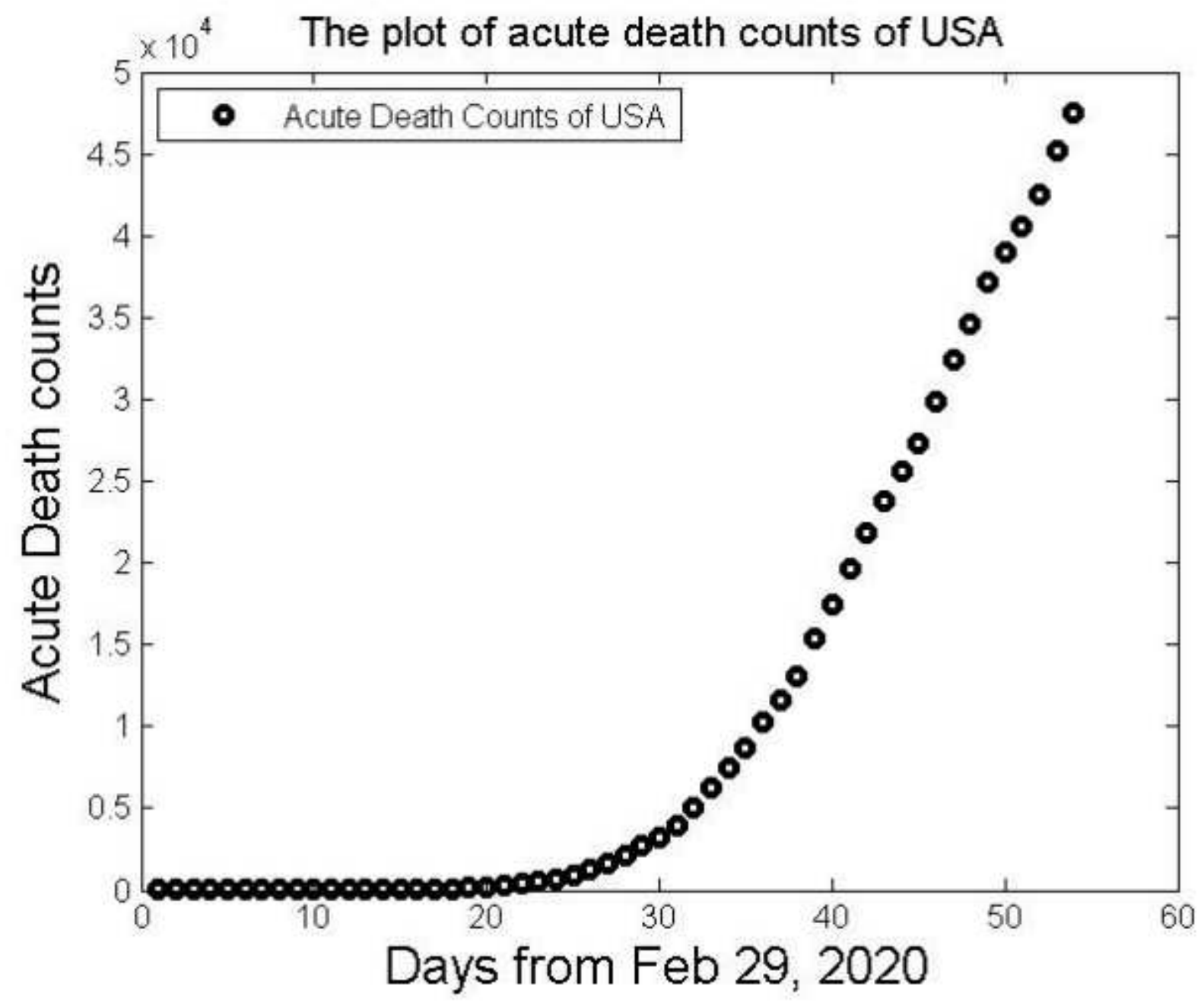

Figure 3

The plot of the Data set of mass acute deaths in USA due to COVID-19 virus . 


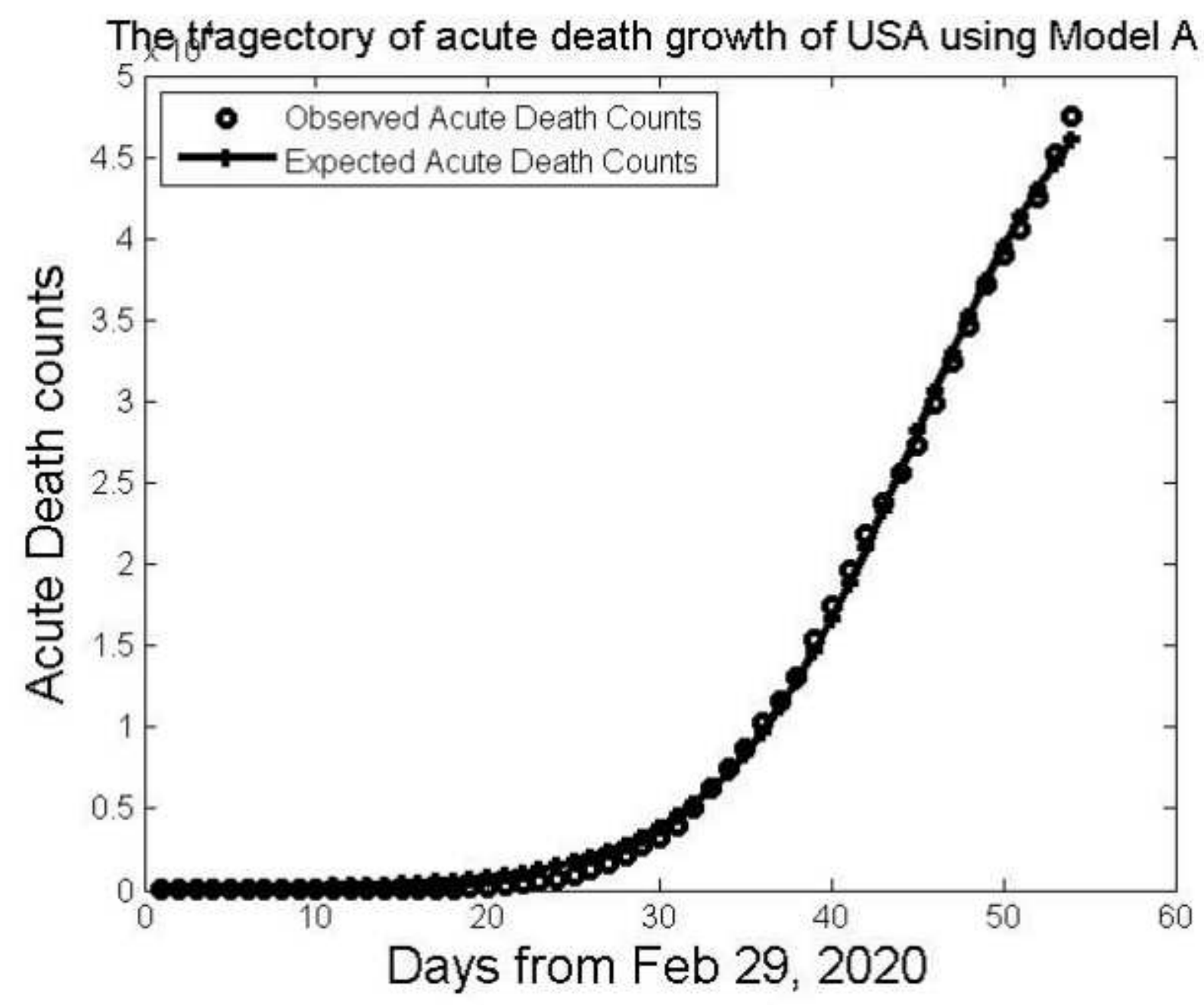

Figure 4

The mass acute death growth trajectory of USA using the mathematical model, Model-A 
The thajectory of acute death growth of USA using Model B

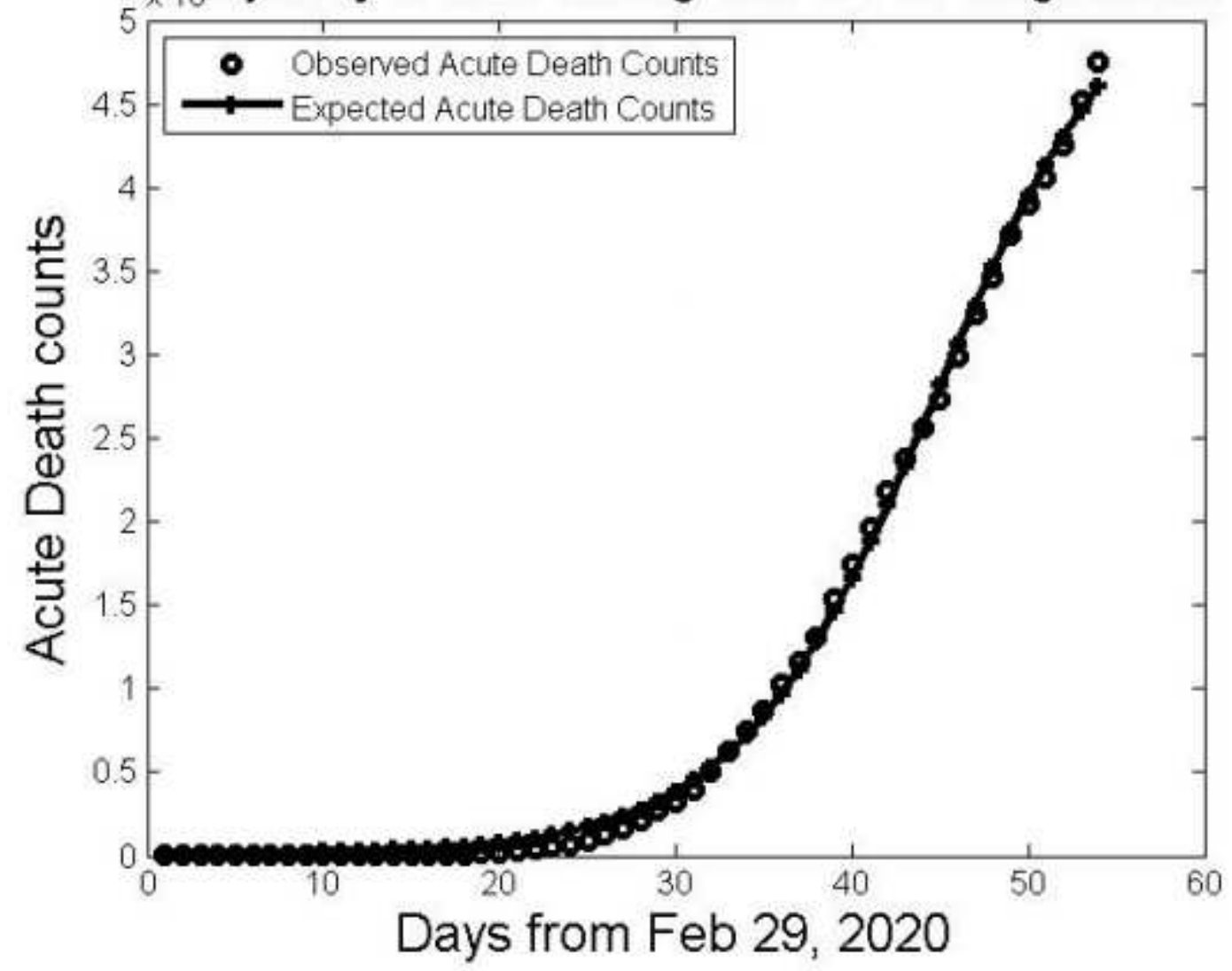

Figure 5

The mass acute death growth trajectory of USA using the mathematical model, Model-B 


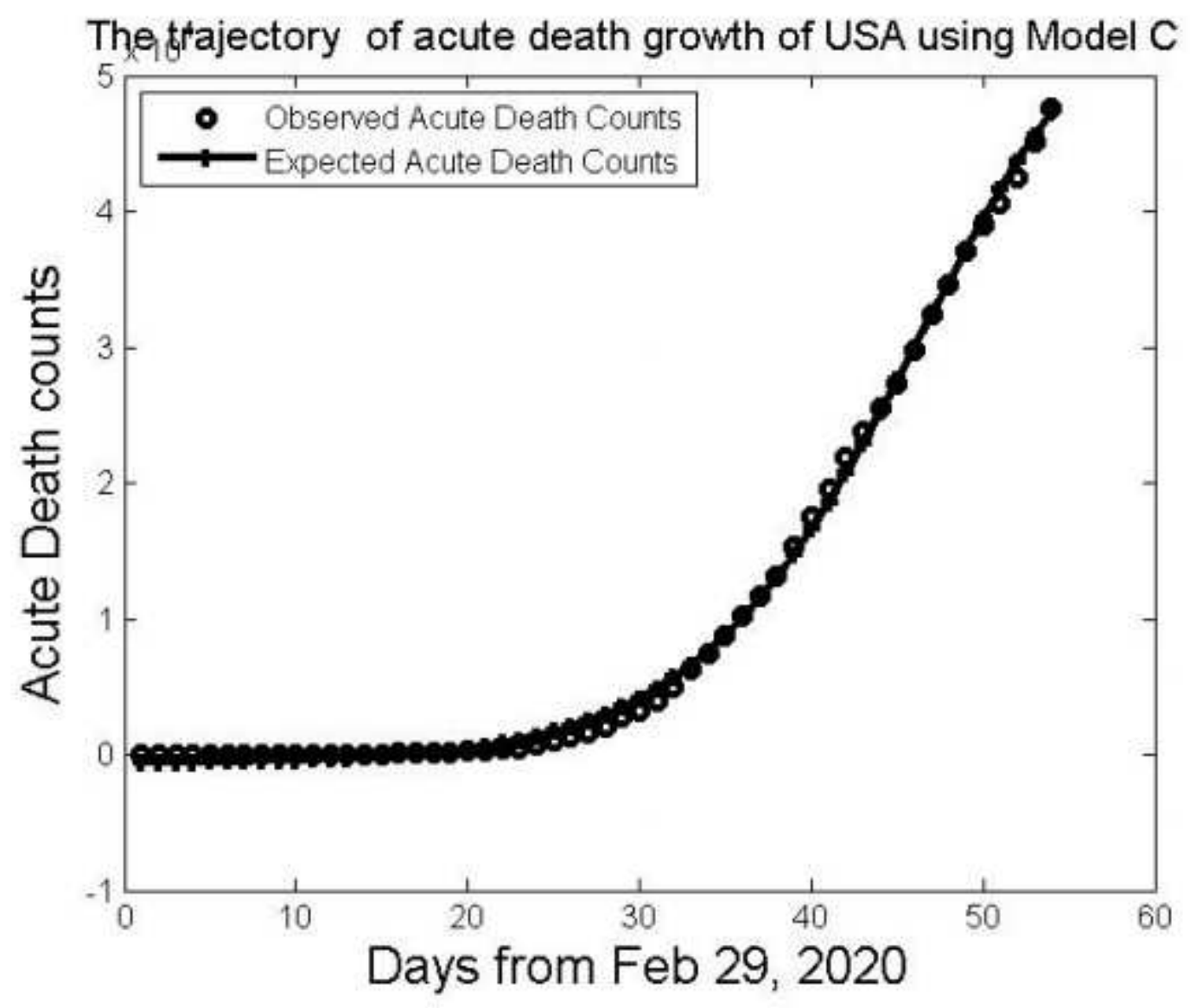

Figure 6

The mass acute death growth trajectory of USA using the mathematical model, Model-C 